\title{
Topological fixed point theory and applications to variational inequalities
}

\author{
Abdul Latif ${ }^{1}$ and Hichem Ben-El-Mechaiekh ${ }^{2 *}$
}

To the memory of George Isac

\author{
"Correspondence: \\ hmechaie@brocku.ca \\ ${ }^{2}$ Department of Mathematics and \\ Statistics, Brock University, Saint \\ Catharines, Ontario L2S 3A1, Canada \\ Full list of author information is \\ available at the end of the article
}

\begin{abstract}
This is the first part of a work on generalized variational inequalities and their applications in optimization. It proposes a general theoretical framework for the solvability of variational inequalities with possibly non-convex constraints and objectives. The framework consists of a generic constrained nonlinear inequality $(\exists \hat{u} \in \Psi(\hat{u})$, $\exists \hat{y} \in \Phi(\hat{u})$, with $\varphi(\hat{u}, \hat{y}, \hat{u}) \leq \varphi(\hat{u}, \hat{y}, v), \forall v \in \Psi(\hat{u}))$ derived from new topological fixed point theorems for set-valued maps in the absence of convexity. Simple homotopical and approximation methods are used to extend the Kakutani fixed point theorem to upper semicontinuous compact approachable set-valued maps defined on a large class of non-convex spaces having non-trivial Euler-Poincaré characteristic and modeled on locally finite polyhedra. The constrained nonlinear inequality provides an umbrella unifying and extending a number of known results and approaches in the theory of generalized variational inequalities. Various applications to optimization problems will be presented in the second part to this work to be published ulteriorly.

MSC: Primary 49J40; 47H10; 47N10; secondary 47H04; 47H19; 49J24

Keywords: constrained nonlinear inequality; variational and quasi-variational inequalities; variational-like inequalities; complementarity problems; approximation methods in set-valued analysis; fixed points; equilibria and co-equilibria for set-valued maps; control problems; differential inclusions; quasiconvex programming; normal and tangent cones
\end{abstract}

\section{Introduction}

The theory of variational inequalities was initiated to study equilibrium problems in contact mechanics. Fichera's treatment of the existence and uniqueness for the Signorini problem in 1962-1963 is viewed as the birth of the theory (see [1] for a first hand historical account). In that problem, at equilibrium, contact points between an elastic body and a rigid surface must satisfy the equilibrium equations in addition to a set of boundary conditions expressed as equalities (on the free boundary of the elastic body) together with inequalities involving displacement and tension along tangent and normal directions to the contact boundary of the body. The analyses of the problem by both Signorini and Fichera were based on a crucial variational argument, namely that the solution of the equilibrium problem ought to be the displacement configuration $\hat{u}$ minimizing the total elastic potential energy functional $I(u)$ amongst admissible displacements $u$. Naturally, such a minimizer must solve the variational inequality $\left.\frac{d}{d t} I(u+t v)\right|_{t=0} \geq 0$ for all admissible directions $v{ }^{\text {a }}$

(C) 2015 Latif and Ben-El-Mechaiekh. This article is distributed under the terms of the Creative Commons Attribution 4.0 International License (http://creativecommons.org/licenses/by/4.0/), which permits unrestricted use, distribution, and reproduction in any medium, provided you give appropriate credit to the original author(s) and the source, provide a link to the Creative Commons license, and indicate if changes were made. 
The directional variation $\left.\frac{d}{d t} I(u+t v)\right|_{t=0}$ takes on the form of a superposition $a(u, v)-F(v)$ of a bilinear form and a linear functional defined on admissible displacements in an appropriate Hilbert space (namely a Sobolev space $H^{1}$ ). The functional analytic framework for the use of variational inequalities as a tool for solving boundary value problems owes much to the pioneering work of Stampacchia. The celebrated existence and uniqueness theorem of Stampacchia (1964) remains a corner stone of the theory in normed spaces of any dimension (see [2]). It intimately links variational inequalities to the minimization of energy functionals and states as follows: given a closed convex subset $X$ of a reflexive (real, for simplicity) Banach space $E$ and a continuous coercive bilinear form $a(\cdot, \cdot)$ on $E \times E$, then

$$
\forall p \in E^{\prime}, \exists ! \hat{u} \in X, \quad a(\hat{u}, v-\hat{u})-p(v-\hat{u}) \geq 0, \quad \forall v \in X .
$$

If in addition $a(\cdot, \cdot)$ is symmetric, then $\hat{u}$ is characterized by $I(\hat{u})=\min _{v \in X} I(v)$, where $I(v)=\frac{1}{2} a(v, v)-p(v)$.

For the Signorini problem, the variational inequality (1) corresponds to the EulerLagrange necessary condition expressing stationarity in the Hamilton principle for the minimization of the energy $I(v)$. Fixed point arguments are at the heart of $(1)$ in more than one respect. On one hand, it can be derived from the Banach contraction principle (see, e.g., [3]). Indeed, the bilinear continuous and coercive $\mathrm{e}^{\mathrm{b}}$ bilinear form $a(\cdot, \cdot)$ defines an inner product whose norm $\|u\|_{a}=a(u, u)^{1 / 2}$ is equivalent to the original norm on $E$. By the Riesz-Fréchet representation theorem, we may write $p(v-\hat{u})=a(p, v-\hat{u})$ with $p \in E$ and view (1) as $\forall p \in E, \exists ! \hat{u} \in X, a(\lambda p-\lambda \hat{u}+\hat{u}-\hat{u}, v-\hat{u}) \leq 0, \forall v \in X$ for any given scalar $\lambda>0$. This formulation is equivalent to a fixed point problem $\hat{u}=P_{X}\left(\lambda p+(1-\lambda) I d_{X}\right)(\hat{u})$ for the orthogonal projection $P_{X}$ onto $X$. The operator $T(v)=P_{X}(\lambda p+(1-\lambda) v)$ is a contraction whenever the scalar $\lambda$ is chosen so that $0<\lambda<2 \alpha / C$. The Banach contraction principle applies to yield the solution's existence and uniqueness. This point of view highlights the intimate relationship between variational inequalities and minimization problems. For applications of contraction principles to variational relations, the reader is referred to [4].

On the other hand, one may adopt an alternate fixed point approach via set-valued analysis - ultimately calling upon the Brouwer theorem or some of its topological generalizations. ${ }^{\mathrm{c}}$ This is the approach adopted in this work in order to study variational inequalities in the presence of non-convexity.

To set the tone, let us note that (1) could alternately and quite easily be obtained as a consequence of two distinct fundamental topological fixed point principles for set-valued maps. The first approach uses the Browder-Ky Fan fixed point theorem (which is equivalent to the Knaster-Kuratowski-Mazurkiewicz-Ky Fan principle) much as in [5] and relies heavily on convexity. Here, the point-to-set map $\Phi: X \rightrightarrows X, \Phi(u):=\{v: a(u, v-u)-p(v-u)<0\}$ turns out to be a so-called Ky Fan map without fixed points on a bounded closed convex subset of $X .^{\mathrm{d}}$ It must have a 'maximal element' $\hat{u}$ with $\Phi(\hat{u})=\emptyset$, i.e., $\hat{u}$ solves (1) (the uniqueness follows at once from the additivity and the coercivity of the form $a$ ). The reader is referred to the early work by Minty [6], to Dugundji-Granas [7] for pioneering the KKM maps approach, ${ }^{\mathrm{e}}$ to Allen [8] for an early concise account and to Lassonde [9] for a comprehensive treatment based on KKM theory.

The second approach is based on a generalization of the Kakutani fixed point theorem much as in Ben-El-Mechaiekh-Isac [10]. This is the point of view we shall focus on here.

In geometric terms of convex analysis, (1) can be written as an orthogonality property $p-\hat{u} \in N_{X}(\hat{u})$, where $N_{X}(\hat{u})$ is the normal cone to $X$ at $\hat{u}$ in the sense of convex analysis. 
Indeed, viewing $E$ as a Hilbert space with inner product $a(\cdot, \cdot),(1)$ amounts to $p-\hat{u} \in$ $(X-\hat{u})^{-}$the negative polar cone of $X-\hat{u}$. But $(X-\hat{u})^{-}=\left(\bigcup_{t>0} \frac{1}{t}(X-\hat{u})\right)^{-}=T_{X}(\hat{u})^{-}=N_{X}(\hat{u})$, where $T_{X}(\hat{u})=\operatorname{cl}\left(\bigcup_{t>0} \frac{1}{t}(X-\hat{u})\right)$ is the tangent cone to $X$ at $\hat{u}$. Thus, (1) can be seen as a set-valued fixed point problem $\hat{u} \in \Psi_{p}(\hat{u}):=p-N_{X}(\hat{u})$ or, equivalently, as an equilibrium (or a zero) problem

$$
0 \in \Phi_{p}(\hat{u})
$$

for the set-valued map $\Phi_{p}: X \rightrightarrows E$ defined as $\Phi_{p}(u):=p-\left(u+N_{X}(u)\right)$. Observe first that if $p \notin X$, then $\hat{u}=P_{X}(p)$ verifies $a(p-\hat{u}, v-\hat{u}) \leq 0, \forall v \in X$, i.e., $p-\hat{u} \in(X-\hat{u})^{-}=N_{X}(\hat{u})$ which amounts to $0 \in p-\hat{u}-N_{X}(\hat{u})=\Phi_{p}(\hat{u})$ and (1) is solved. If $p \in X$, then $p \in u+T_{X}(u), \forall u \in X$, thus $p-u=p-u+0_{E} \in\left(p-u-N_{X}(u)\right) \cap T_{X}(u)$. Generalizations to infinite dimensions of the Bolzano-Poincaré intermediate value theorem (see, e.g., Ben-El-Mechaiekh [11]) can be used to solve (2) as the map $\Phi_{p}$ has closed convex values, is upper semicontinuous, and satisfies the tangency boundary condition $\Phi(u) \cap T_{X}(u) \neq \emptyset, \forall u \in X$. This approach lends itself to the treatment of non-convex problems through the consideration of natural and appropriate topological substitutes to convexity as well as corresponding notions of tangency from non-smooth analysis.

We have briefly described above the intimate relationships between the Stampacchia variational inequality (1), minimization problems, general nonlinear inclusions, and fixed point principles. The theory of variational inequalities is playing an increasingly central role in the study of problems not only in mechanics, physics, and engineering but also in optimization, game theory, finance, economics, population dynamics, etc. The theory has vastly expanded in the past five decades with the intensive production of literature on numerous functional analytic, qualitative, and computational aspects. The interested reader is referred to the books by Baiocchi and Capelo [12], Kinderlehrer and Stampacchia [13], Nagurney [14], Granas [15], Cottle et al. [16], Isac [17], Murty [18], Facchinei and Pang [19], Konnov [20], Ansari et al. [21], as well as the papers by Gwinner [22], Blum and Oettli [23], Agarwal and O'Regan [24] and recently the survey paper by Ansari [25].

This paper is the first part of a work devoted to the study of generalized variational inequalities on non-convex sets. It describes the constrained inequalities umbrella framework for variational and quasi-variational inequalities. The main existence results on general systems of constrained inequalities (Theorems 17, 20 below) are derived from new topological generalizations of the fixed point theorem of Kakutani without convexity (Theorems 12 and 15). The domains considered are spaces modeled on locally finite polyhedra having non-trivial Euler-Poincaré characteristic which are not necessarily compact. Rather, compactness is imposed on the maps. Solvability of generalized variational inequalities expressed as co-equilibria problems for non-self non-convex set-valued maps defined on Lipschitzian retracts is established in the last section (Theorem 32 and Corollary 33). The paper also illustrates how the general results apply to particular situations in the theories of variational inequalities, complementarity, and optimal control.

\section{A general constrained nonlinear inequality}

We assume that vector spaces are over the real number field and topological vector spaces are Hausdorff. Set-valued maps (simply called maps) are denoted by capital letters and double arrows $\rightrightarrows$. 
Given two non-empty sets $X, Y$, two maps $\Psi: X \rightrightarrows X, \Phi: X \rightrightarrows Y$, and a proper real function $\varphi: X \times Y \times X \rightarrow(-\infty,+\infty]$, consider the constrained nonlinear inequality

$$
\left\{\begin{array}{l}
\exists \hat{u} \in \Psi(\hat{u}), \exists \hat{y} \in \Phi(\hat{u}), \text { with } \\
\varphi(\hat{u}, \hat{y}, \hat{u}) \leq \varphi(\hat{u}, \hat{y}, v), \forall v \in \Psi(\hat{u}) .
\end{array}\right.
$$

The solvability results for CNI will be discussed in Section 4. For now, let us make clear that the CNI framework includes several types of variational inequalities.

- Generalized quasi-variational inequalities

Given a vector space $E$ and a dual pair $\left\langle F^{*}, F\right\rangle$ of vector spaces, ${ }^{\mathrm{f}}$ given two non-empty subsets $X \subseteq E, Y \subseteq F$ and two maps $\Psi: X \rightrightarrows X, \Phi: X \rightrightarrows Y$, given two mappings $\theta: X \times$ $Y \rightarrow F^{*}, \eta: X \times X \rightarrow F$ and a functional $\phi: X \rightarrow \mathbb{R}$, the generalized quasi-variational inequality problem QVI associated to the data $(X, Y, \Psi, \Phi, \theta, \eta, \phi)$ is

$$
\left\{\begin{array}{l}
\exists \hat{u} \in \Psi(\hat{u}), \exists \hat{y} \in \Phi(\hat{u}) \text { with } \\
\langle\theta(\hat{u}, \hat{y}), \eta(v, \hat{u})\rangle \geq \phi(\hat{u})-\phi(v), \forall v \in \Psi(\hat{u}) .
\end{array}\right.
$$

Obviously, the existence part in the variational inequality (1) is a very particular case of QVI - hence of CNI - whereby $E=F$ is a reflexive Banach space that is Hilbertisable by the bilinear continuous and coercive form $a(\cdot, \cdot)$ [thus $E$ identifies with its topological dual $E^{\prime}$, the dual pairing being obviously $\langle p, u\rangle=a(p, u)]$, and $X=Y \subseteq E, \Phi(u)=\Psi(u)=X$ for all $u$ are constant maps, $\theta(u, y)=u$ for all $y, \eta(v, u)=v-u$, and $\phi=-p$.

In fact, quite clearly, QVI contains the so-called variational-like inequalities of the Stampacchia type: given a dual pair of vector spaces $\langle E, F\rangle$, a non-empty closed subset $X \subseteq E$, a set-valued map $\Phi: X \rightrightarrows F$, and a mapping $\eta: X \times X \rightarrow E$,

$$
\exists \hat{u} \in \Psi(\hat{u}), \exists \hat{y} \in \Phi(\hat{u}) \quad \text { with }\langle\hat{y}, \eta(v, \hat{u})\rangle \geq 0, \forall v \in X .
$$

This problem is clearly equivalent to the equilibrium problem

$$
0 \in \Phi(\hat{u})+\eta(X, \hat{u})^{-},
$$

where $\eta(X, u)^{-}=\{y \in F:\langle\hat{y}, \eta(v, u)\rangle \leq 0$ for all $v \in X\}$ is a negative polar cone.

In case $\eta(v, u)=v-u$, then $\eta(X, u)^{-}=(X-u)^{-}=\left(\bigcup_{t>0} \frac{1}{t}(X-\hat{u})\right)^{-}$. If in addition $E$ is equipped with the weak topology $\sigma(E, F)$ - or with any topology for which the linear forms $x \mapsto\langle y, x\rangle, y \in F$, are continuous on $E$ - then $\eta(X, u)^{-}=N_{X}^{R}(\hat{u})=T_{X}^{R}(\hat{u})^{-}$, where $T_{X}^{R}(u)=$ $\operatorname{cl}\left(\bigcup_{t>0} \frac{1}{t}(X-\hat{u})\right)$ is the radial cone (which is simply the tangent cone of convex analysis $T_{X}(\hat{u})$ whenever $X$ is locally convex at $\left.\hat{u}\right)$. Thus, (3) writes

$$
0 \in \Phi(\hat{u})+N_{X}^{R}(\hat{u}) .
$$

The latter inclusion, referred to as a generalized variational inequality, covers (2) whenever $E=F$ and $\Phi(u)=-p+u$ is single-valued. This case strongly relates to the minimization of functionals as described next.

- Quasiconvex programming

It is well established that for a proper Gâteaux-differentiable (on its effective domain, assumed to be open and convex) function $f: E \rightarrow(-\infty,+\infty]$ of a topological vector space $E$, quasi-convexity ${ }^{g}$ is equivalent to the proposition [given $\left.u, v \in X=\operatorname{dom}(f),\langle\nabla f(u), v-u\rangle\right\rangle$ 
$0 \Longrightarrow f(u) \leq f(v)]$ - see, e.g., Proposition 4.12 in [26]. Thus, the strict ${ }^{\text {h }}$ variational inequality $\exists \hat{u} \in X,\langle\nabla f(\hat{u}), v-\hat{u}\rangle>0$ implies that $f(\hat{u})=\min _{X} f(v)$.

This characterization of quasi-convexity extends to a non-smooth function. Indeed, if $f$ is only lower semicontinuous (not necessarily differentiable) on its effective domain $X$, and if the space $E$ is a Banach space with a Gâteaux differentiable (equivalent) norm, then quasi-convexity is equivalent to

$\exists p \in \partial f(u) \quad$ such that $\langle p, v-u\rangle>0 \Longrightarrow f(w) \leq f(v), \quad \forall w \in[u, v[$,

where $\partial f(u)$ is the lower Dini subdifferential of $f$ at $u$. Obviously, if the special instance of (VIS)

$\exists \hat{u} \in X, \exists \hat{p} \in \partial f(\hat{u}) \quad$ such that $\langle\hat{p}, v-\hat{u}\rangle>0, \quad \forall v \in X$,

holds for a quasiconvex and lower semicontinuous function $f$, then $f(\hat{u})=\min _{X} f(v)$.

As pointed out by Aussel [27], a better adapted approach to quasiconvex programming relies on the so-called adjusted normal cone to sublevel sets. Consider the sublevel set operators $S_{f<}, S_{f \leq}: X \rightrightarrows X$ defined by $S_{f \leq}(u):=\{y \in X: f(y) \leq f(u)\}$ and $S_{f<}:=\{y \in X:$ $f(y)<f(u)\}$, where $X$ is the effective domain of $f: E \rightarrow(-\infty,+\infty], E$ being a metrizable topological vector space. Clearly, $S_{f<}$ has non-empty values at points $u \in X \backslash \arg \min f$ and is a selector of $S_{f \leq}$. Let $\rho_{u}=d\left(u, S_{f<}(u)\right)$ and consider, for each $u \in X$, the adjusted level set

$$
S_{f}^{a}(u):= \begin{cases}S_{f \leq}(u) \cap \bar{B}\left(S_{f<}(u), \rho_{u}\right) & \text { if } u \notin \arg \min _{X} f, \\ S_{f \leq}(u) & \text { else. }\end{cases}
$$

Always, $S_{f<}(u) \subseteq S_{f}^{a}(u) \subseteq S_{f \leq}(u)$ for all $u \in X$; if $f$ is l.s.c., then $S_{f<}(u) \subseteq \operatorname{cl}\left(S_{f<}(u)\right) \subseteq S_{f}^{a}(u) \subseteq$ $S_{f \leq}(u)$ and at points $u$ with $\rho_{u}=0$, we have $\operatorname{cl}\left(S_{f<}(u)\right)=S_{f}^{a}(u)$. All four sublevel set operators $S_{f<}(\cdot), \operatorname{cl}\left(S_{f<}(\cdot)\right), S_{f}^{a}(\cdot)$, and $S_{f \leq}(\cdot)$ have convex values if and only if $f$ is quasiconvex. The adjusted normal cone operator $N_{f}^{a}: X \rightrightarrows E^{\prime}$ is defined as the polar cone:

$$
N_{f}^{a}(u):= \begin{cases}\left(S_{f}^{a}(u)-u\right)^{-} & \text {if } u \in X \backslash \arg \min _{X} f, \\ \left(S_{f \leq}(u)-u\right)^{-} & \text {if } f(u)=\min _{X} f .\end{cases}
$$

Denoting the negative polar cones of $S_{f<}(u)-u$ and $S_{f \leq}(u)-u$ by $N_{f<}(u)$ and $N_{f \leq}(u)$, respectively, we have $N_{f \leq}(u) \subseteq N_{f}^{a}(u) \subseteq N_{f<}(u)$ for all $u \in X$, with all sets being closed convex cones. If $f$ is quasiconvex, then for all $u \in X \backslash \arg \min _{X} f$, the set $N_{f}^{a}(u)$ has non-trivial elements. Proposition 5.16 in [27] provides a sufficient optimality condition for quasiconvex programming: for a quasiconvex function $f: E \rightarrow(-\infty,+\infty]$ radially continuous on its effective domain $X$, given a (not necessarily convex) subset $C \subseteq \operatorname{int}(X)$, if a special instance of (VIS) is solvable over $C$ with $\Phi=N_{f}^{a} \backslash\{0\}$ (or $N_{f<} \backslash\{0\}$, obviously) and $\eta(v, u)=v-u$, i.e., if $\exists \hat{u} \in C, \exists \hat{y} \in N_{f}^{a}(\hat{u}) \backslash\{0\}$ such that $\langle\hat{y}, v-\hat{u}\rangle \geq 0$ for all $v \in C$, then $f(\hat{u})=\inf _{C} f$. Taking (4) into account, we have

$$
\exists \hat{u} \in C \quad \text { with } 0 \in\left(N_{f}^{a}(\hat{u}) \backslash\{0\}\right)+N_{C}^{R}(\hat{u}) \quad \Longrightarrow \quad f(\hat{u})=\inf _{C} f .
$$

Conversely, if $C$ is a closed convex subset of the effective domain of a semistrictly quasiconvex $^{\mathrm{i}}$ continuous function $f: E \rightarrow(-\infty,+\infty]$ such that $f(\hat{u})=\inf _{C} f$ and $\operatorname{int}\left(S_{f \leq}(\hat{u})\right) \neq \emptyset$, then $0 \in\left(N_{f}^{a}(\hat{u}) \backslash\{0\}\right)+N_{C}^{R}(\hat{u})$ (here, $N_{C}^{R}$ is the normal cone of convex analysis). 


\section{- Multivalued complementarity problems}

Multivalued complementarity problems are also very particular cases of QVI. Recall that given a dual pair of vector spaces $\langle F, E\rangle$ and a cone $X \subset E$ with dual cone $X^{*}=\{y \in$ $F ;\langle y, x\rangle \geq 0, \forall x \in X\}$, and given a set-valued map $\Phi: X \rightrightarrows F$, a mapping $f: X \times F \rightarrow F$ and a functional $\phi: X \rightarrow \mathbb{R}$, the multivalued complementarity problem (associated to $(X, \Phi, f, \phi))$ is

$$
\left\{\begin{array}{l}
\exists \hat{u} \in X, \exists \hat{y} \in \Phi(\hat{u}) \cap X^{*} \text { such that } \\
f(\hat{u}, \hat{y}) \in X^{*} \text { and }\langle f(\hat{u}, \hat{y}), \hat{u}\rangle=\phi(\hat{u}) .
\end{array}\right.
$$

The classical generalized multivalued complementarity problem corresponds to $\phi(u)$ being identically zero and $f(u, y)=y$ (see, e.g., [17]).

- A general optimal control problem

Let $I=$ interval in $\mathbb{R}, X$ closed $\subset E$ separable Banach space, $F: I \times X \rightrightarrows E, S_{F}(u)=$ solutions viable in $X$ for the Cauchy problem

$$
\left\{\begin{array}{l}
y^{\prime}(t) \in F(t, y(t)), \quad t \in I, y(t) \in X \\
y\left(t_{0}\right)=u \in X,
\end{array}\right.
$$

(assuming such solutions exist). Starting at a point $u \in X$, consider the journey along a trajectory $y(t)$ of (6) followed by a path to a point $v$ in a return set $\Psi(u)$. Assume that a cost $\varphi(u, y, v)$ is associated to this journey $\left(e . g ., \varphi(u, y, v)=\varphi_{1}(u, y)+\varphi_{2}(y, v)\right)$. We will discuss in Section 5 the case of the particular instance of CNI, namely the general optimal control problem

$$
\left\{\begin{array}{l}
\exists \hat{u} \in X, \hat{u} \in \Psi(\hat{u}), \exists \hat{y} \in S_{F}(\hat{u}) \text { such that } \\
\varphi(\hat{u}, \hat{y}, \hat{u})=\inf _{v \in \Psi(\hat{u})} \varphi(\hat{u}, \hat{y}, v) .
\end{array}\right.
$$

\section{Fixed points without convexity}

The main general existence results for constrained nonlinear inequalities of this paper (Theorems 17, 20 below) derive from new fixed point theorems for approachable setvalued maps in the sense on Ben-El-Mechaiekh-Deguire ([28]; see also [29]) defined on spaces modeled over locally finite polyhedra, in particular ANRs (Theorems 12, 15 and Corollaries 16, 33). Before getting to the fixed point and equilibrium results, we briefly recall fundamental topological concepts used as a substitute for convexity together with the definition, examples, and properties of approachable maps.

\subsection{Approachable maps on ANRs}

Definition $1([5])$ Let $(X, \mathcal{U})$ and $(Y, \mathcal{V})$ be two topological spaces with compatible uniformity structures $\mathcal{U}$ and $\mathcal{V}$. A map $\Phi: X \rightrightarrows Y$ is said to be approachable if and only if, for each entourage $W$ of the product uniformity $\mathcal{U} \times \mathcal{V}$ on $X \times Y$, there exists a continuous single-valued mapping $s: X \rightarrow Y$ satisfying the inclusion $\operatorname{graph}(s) \subset W[\operatorname{graph}(\Phi)]$.

Thus, approachable maps are those maps admitting arbitrarily close single-valued continuous graph approximations, also known as continuous approximative selections. Indeed, it is easy to see that $\Phi$ is approachable if and only if $\forall U \in \mathcal{U}, \forall V \in \mathcal{V}$, $\Phi$ admits 
a continuous $(U, V)$-approximative selection, i.e., a continuous single-valued function $s: X \rightarrow Y$ verifying

$$
s(x) \in V[\Phi(U[x])], \quad \forall x \in X .
$$

This continuous graph approximation property turns out to be, in presence of some compactness, a by-product of the upper semi-continuity of the map $\Phi$ together with a qualitative topological/geometric property of its values. The classical convex example (which can be traced back to von Neumann's proof of its famous minimax theorem) is a case in point.

Example 2 (Convex case, [30]) Let $X$ be a paracompact topological space equipped with a compatible uniformity $\mathcal{U}$, and let $Y$ be a convex subset of a locally convex topological vector space $F$. Let $\Phi: X \rightrightarrows Y$ be an upper semicontinuous' (u.s.c. for short) map with non-empty convex values. Then $\Phi$ is approachable.

This landmark result has been extended to natural topological notions extending convexity which we consider in this work. Recall that a topological space $X$ is said to be contractible (in itself) if there exist a fixed element $x_{0} \in X$ and a continuous homotopy $h: X \times[0,1] \rightarrow X$ such that $h(x, 0)=x$ and $h(x, 1)=x_{0}, \forall x \in X$. Clearly, every convex and, more generally, every star-shaped subset of a topological vector space is contractible.

Absolute retracts are important examples of contractible spaces and occupy a central place in topological fixed point theory as initiated by Karol Borsuk. We recall here basic facts on retracts that are crucial for the sequel. For a detailed exposition on absolute retracts and absolute neighborhood retracts (ARs and ANRs for short), we refer to the book of Jan Van Mill [31].

\section{Definition 3}

(i) A subspace $A$ of a topological space $X$ is a neighborhood retract of $X$ if some open neighborhood of $A$ in $X$ can be continuously retracted into $A$, i.e., there exist an open neighborhood $V$ of $A$ in $X$ and a continuous mapping $r: V \rightarrow A$ such that $r(a)=a$ for all $a \in A$. If $V=X, A$ is simply said to be a retract of $X{ }^{\mathrm{k}}$

(ii) A metric space $A$ is an absolute (neighborhood) retract - written $A \in A R(A \in A N R$, resp.) - if and only if $A$ is an absolute (neighborhood) retract of every metric space in which it is imbedded.

(iii) A metric space $A$ is an approximative absolute neighborhood retract $(A \in A A N R$ for short) if and only if $A$ is an approximative neighborhood retract of any metric space $(X, d)$ in which it is imbedded as a closed subspace; i.e., for any $\epsilon>0$, there exists an open neighborhood $V$ of $A$ in $X$ and a continuous mapping $r: V \rightarrow A$ such that $d(r(a), a)<\epsilon$ for all $a \in A$.

Note that $\mathrm{AR} \subset \mathrm{ANR} \subset \mathrm{AANR} .{ }^{1}$ Observe also that if $A$ is a retract of a topological space $X$ with retraction $r: X \rightarrow A$, then any continuous mapping $f_{0}: A \rightarrow Y$ into any topological space $Y$ extends to the continuous mapping $f=r \circ f_{0}: X \rightarrow A \rightarrow Y$. Thus, retracts and neighborhood retracts are characterized by extension properties. In effect, every AR is an absolute extensor for metric spaces. This implies that each AR is contractible in itself. ${ }^{\mathrm{m}}$ Also, every AR is a retract of some convex subspace of a normed linear space. Conversely, 
the Dugundji extension theorem (see, e.g., [7] or [5]) asserts that convex sets in locally convex spaces are absolute extensors for metric spaces. Hence, any metrizable retract of a convex subset of a locally convex topological linear space is an AR. Every infinite polyhedron endowed with a metrizable topology is an AR. Similarly, every ANR is an absolute neighborhood extensor of metric spaces. Even more precisely, ANRs are characterized as retracts of open subsets of convex subspaces of normed linear spaces. The class ANR include all compact polyhedra. Every Fréchet manifold is an ANR. The union of a finite collection of overlapping closed convex subsets in a locally convex space is an ANR provided it is metrizable (see [31]). AANRs as characterized as metrizable spaces that are homeomorphic to approximative neighborhood retracts of normed spaces.

We now state some extensions of Example 2 to maps with non-convex values. We start with the contractible case.

Example 4 (Contractible case, [28, 32, 33]) Given two ANRs $X$ and $Y$ with $X$ compact, every u.s.c. map $\Phi: X \rightrightarrows Y$ with compact contractible values is approachable.

Contractibility is not sufficient to describe qualitative properties of solution sets to some differential or integral equations and inclusions. A seminal result of Aronszajn [34] establishes that such solution sets satisfy a more general proximal contractibility property tantamount to being contractible in each of their open neighborhoods (sets with trivial shape and $R_{\delta}$ sets $\left.^{\mathrm{n}}\right)$. To be more precise, let us consider the following notion.

Definition 5 (Dugundji [35]) A subspace $Z$ of a topological space $Y$ is said to be $\infty$ proximally connected in $Y$ if for each open neighborhood $U$ of $Z$ in $Y$, there exists an open neighborhood $V$ of $Z$ in $Y$ contained and contractible in $U$.

\section{Example 6}

(i) The set $\left\{\left(t, \sin \left(\frac{1}{t}\right)\right) ; 0<t \leq 1\right\} \cup(\{0\} \times[-1,1])$ is not contractible in itself, but it is contractible in each of its open neighborhoods in $\mathbb{R}^{2}$.

(ii) If a subspace $Z$ of an ANR $Y$ has trivial shape in $Y$ (that is, $Z$ is contractible in each of its neighborhoods in $Y$ ), then $Z$ is $\infty$-proximally connected in $Y$ (see [31]).

(iii) Let $\left\{Z_{i}\right\}_{i=1}^{\infty}$ be a decreasing sequence of compact spaces having trivial shape in an ANR $Y$. Then $Z=\bigcap_{i=1}^{\infty} Z_{i}$ is $\infty$-proximally connected in $Y$ (see [35]). In particular, every $R_{\delta}$ set in an ANR $Y$ is $\infty$-proximally connected in $Y$.

We now state extensions of Example 4 to maps with non-contractible values.

Example 7 (Non-contractible cases)

(i) (Compact domains, $[28,29]$ ) Let $X$ be a compact AANR and let $Y$ be a uniform space. Then every u.s.c. map $\Phi: X \rightrightarrows Y$ with non-empty compact $\infty$-proximally connected values in $Y$ is approachable.

(ii) (Non-compact domains, [36]) Let $X$ be an ANR and let $Y$ be a metric space. Then every u.s.c. map $\Phi: X \rightrightarrows Y$ with non-empty compact $\infty$-proximally connected values in $Y$ is approachable.

Case (i) is a particular version of a result in [29] (see Corollary 2.17 there or Corollary 3.4 in [5]), where the non-metrizable case - $X$ is an approximative absolute neighborhood 
extension space (AANES) for compact topological spaces - is considered (compact AANRs are AANES for compact spaces). In the special case where $X$ and $Y$ are ANRs with $X$ compact, this result first appeared in [37].

Examples 2, 4, and 7 indicate that some compactness of the domain plays a key role in the approachability of a map (ANRs are paracompact spaces). Compactness can be weakened by simply requesting approachability on finite polyhedra. More precisely:

Proposition 8 Let $X$ be an $A N R,(Y, \mathcal{V})$ be a uniform topological space, and let $\Phi: X \rightrightarrows$ $Y$ be a u.s.c. map with non-empty values. If the restriction of $\Phi$ to any finite polyhedron $P \subset X$ is approachable on $P$, then the restriction of $\Phi$ to any compact subset $K$ of $X$ is also approachable on $K$.

Proof We only sketch the proof. Recall that given an open subset $U$ of a normed space and a compact subset $K$ of $U$, there exists a compact ANR $C$ such that $K \subset C \subset U$ (Girolo [38]). Since $X$ can be seen as a retract of an open set in a normed space (namely, the space of bounded continuous real functions on $X$ ), one concludes that if $K$ is any compact subset of $X$, then there exists a compact ANR $C$ such that $K \subset C \subset X$. Since compact ANRs are dominated by finite polyhedra and since the enlargement of classes of topological spaces by domination of domain preserves approachability (see Proposition 3.17 in [5]), it follows that the restriction $\left.\Phi\right|_{C}$ of $\Phi$ to $C$ is also approachable. Invoking the fact that restrictions of approachable maps to compact subsets are also approachable (Proposition 3.10 in [5] or Proposition 2.3 in [29]), it follows that the restriction $\left.\Phi\right|_{K}$ of $\Phi$ to $K$ is also approachable.

We now formulate (without proofs) two stability properties for approachable maps essential to the proofs of the main results in Section 3.2 below.

Proposition 9 (See [5]) Given three topological spaces equipped with compatible uniformity structures $(X, \mathcal{U}),(Y, \mathcal{V})$ and $(Z, \mathcal{W})$, let $\Phi: X \rightrightarrows Y$ be a u.s.c. approachable map with non-empty compact values, and let $\Psi: Y \rightrightarrows Z$ be a u.s.c. map with non-empty values such that the restriction of $\Psi$ to the set $\Phi(X)$ is approachable. Then the composition product $\Psi \Phi: X \rightrightarrows Z$ is u.s.c. and approachable provided the space $X$ is compact.

This implies the following.

Example 10 Let $\Phi: X_{0} \rightrightarrows X_{n}$ be a map that admits a decomposition $\Phi(x)=\left(\Phi_{n} \circ \cdots \circ\right.$ $\left.\Phi_{1}\right)(x)$, where each map $\Phi_{i}: X_{i-1} \rightrightarrows X_{i}$ is u.s.c. with $\infty$-proximally connected in an ANR $X_{i}$ for all $i=1, \ldots, n$. Then the restriction of $\Phi$ to each compact subspace of $X_{0}$ is approachable.

\subsection{Fixed point theorems}

The general nonlinear inequality presented in Section 4 below is based on Theorem 15 which is a generalization of the Borsuk and the Eilenberg-Montgomery fixed point theorems to approachable compact set-valued maps defined on spaces dominated by locally finite polyhedra and having non-zero Euler-Poincaré characteristic.

The following observation by the second author [29] provides the essence of the passage from 'almost fixed point' to fixed point for u.s.c. maps. 
Lemma 11 (Lemma 3.1 in [29]) Let $X$ be a regular topological space and $\Gamma: X \rightrightarrows X$ be a u.s.c. map with non-empty closed values. Assume that there exists a cofinal family $\{\omega\}$ of open $($ in $X)$ covers of $K=\mathrm{cl}(\Gamma(X))$ such that $\Gamma$ has an $\omega$-fixed point ${ }^{\circ}$ for each open cover $\omega$. Then $\Gamma$ has a fixed point.

The case of convex domains is much simpler as the next generalization of the fixed point theorems of Ky Fan [39] and Himmelberg [40] to approachable maps shows.

Theorem 12 Let $X$ be a non-empty convex subset of a Hausdorff locally convex space $E$ and $\Gamma: X \rightrightarrows X$ be a u.s.c. map with non-empty closed values such that:

(i) $\Gamma$ is a compact map, i.e., $K=\operatorname{cl}(\Gamma(X))$ is compact in $X$;

(ii) for each finite subset $N$ of $K$, the restriction of the map $\Gamma$ to the convex hull $\operatorname{conv}\{N\}$ of $N$ is approachable.

Then $\Gamma$ has a fixed point.

Given any open convex symmetric neighborhood $U$ of the origin in $E$, the compact set $K$ can be covered by $\bigcup_{i=1}^{n}\left(y_{i}+U\right) \cap X, N=\left\{y_{i} \in K: i=1, \ldots, n\right\}$. The Schauder projection associated to $U, \pi_{U}: \bigcup_{i=1}^{n}\left(y_{i}+U\right) \cap X \rightarrow \operatorname{conv}\{N\}$, verifies

$$
\pi_{U}(y)-y \in U, \quad \forall y \in \bigcup_{i=1}^{n}\left(y_{i}+U\right) \cap X
$$

(note that $\pi_{U}(y)=\frac{1}{\sum_{i=1}^{n} \mu_{i}(y)} \sum_{i=1}^{n} \mu_{i}(y) y_{i}$, where $\mu_{i}(y):=\max \left\{0,1-p_{U}\left(y-y_{i}\right)\right\}, p_{U}$ being the Minkowski functional associated to $U$, is a convex combination). By hypothesis (ii) and Proposition 9, the composition product $\Gamma_{U}=\left.\pi_{U} \circ \Gamma\right|_{\operatorname{conv}\{N\}}: \operatorname{conv}\{N\} \rightrightarrows \bigcup_{i=1}^{n}\left(y_{i}+U\right) \cap$ $X \rightarrow \operatorname{conv}\{N\}$ is approachable. (Note that since $\Gamma(X) \subset K$, any suitable continuous $(V, V)$ approximative $f$ selection of $\left.\Gamma\right|_{\operatorname{conv}\{N\}}(V \subset U)$ has values in the uniform open neighborhood $K+U$ of $K$; thus $\pi_{U} f$ is a continuous approximative selection of $\Gamma_{U}$.) By the extension of Kakutani fixed point theorem to approachable maps (see [28]), $\Gamma_{U}$ has a fixed point $\bar{x}_{U}$ which is a $U$-fixed point for $\Gamma$. As $K$ is compact and $\Gamma$ is $u$.s.c. with compact values, Lemma 11 ends the proof.

Note that if $\Gamma$ is approachable, then its restriction to any compact subset of its domain is also approachable (Proposition 2.3 in [29] for the case of topological vector spaces and Proposition 3.10 in [5] for the general case); thus (ii) always holds true in case $\Gamma$ is approachable.

We turn our attention to the case of the domain being an ANR. It is well established that for any given ANR $X$ and any open cover $\omega$ of $X$, the geometric nerve $|N(\omega)|$ of the cover $\omega$-homotopy dominates $X$ in the following sense: there exist continuous mappings $s: X \rightarrow|N(\omega)|$ and $r:|N(\omega)| \rightarrow X$ such that $r \circ s$ and $\mathrm{id}_{X}$ are $\omega$-homotopic. ${ }^{\mathrm{P}}$ Since ANRs are paracompact, the polyhedron $|N(\omega)|$ is locally finite. This motivates the following definition.

\section{Definition 13}

(i) Given an open cover $\omega$ of a topological space $X$ and a topological space $P$, we say that the space $P \omega$-dominates $X$ ( $\omega_{H}$-dominates $X$, respectively) if there are continuous mappings $s: X \rightarrow P$ and $r: P \rightarrow X$ such that $r \circ s$ and $\operatorname{id}_{X}$ are $\omega$-near ( $\omega$-homotopic, respectively). 
(ii) Given a class of topological spaces $\mathcal{P}$, the classes $D(\mathcal{P})$ and $D_{H}(\mathcal{P})$ of topological spaces dominated and homotopy dominated by $\mathcal{P}$ are defined as: $X \in D(\mathcal{P})$ $\left(X \in D_{H}(\mathcal{P})\right.$, respectively) if and only if $\forall \omega$ open cover of $X, \exists P \in \mathcal{P}$ such that $P$ $\omega$-dominates $X$ ( $\omega_{H}$-dominates $X$, respectively).

Clearly $D_{H}(\mathcal{P}) \subset D(\mathcal{P})$. It is well established that ANRs $\subset D_{H}(\mathcal{P})$, where $\mathcal{P}$ is the class of polyhedra endowed with the CW-topology (see, e.g., Example 3, Section 1.3 in [41]). Compact ANRs as well as $\mathcal{C}$-convex subsets of locally $\mathcal{C}$-convex metrizable topological spaces (where $\mathcal{C}$ is a convexity structure (linear or topological) are dominated by finite polyhedra (see [5])).

We will require the following specialization of the property of domination by locally finite polyhedra for spaces with non-trivial Euler-Poincaré characteristic.

Lemma 14 Let $\mathcal{P}$ be the class of polyhedra and let $X \in D(\mathcal{P})$ be a paracompact space with a well-defined non-trivial Euler-Poincaré characteristic $\mathcal{E}(X) .{ }^{\mathrm{q}}$ Given any open cover $\omega$ of $X$, then there exists a locally finite polyhedron $P$ such that:

(i) $P$ w-homotopy dominates $X$, and

(ii) $\mathcal{E}(P)$ is well defined and non-trivial.

Proof Let $\omega$ be any given locally finite open cover of $X$ and let $|N(\omega)|$ be its geometric nerve. By Lemma 7.2 in [42], since $|N(\omega)|$ is a locally finite polyhedron (thus an $A N R$ ), there exists an open cover $\alpha$ of $|N(\omega)|$ such that any two continuous mappings $f, g: Z \rightarrow|N(\omega)|$ of a space $Z$ that are $\alpha$-near are homotopic. By a theorem of Withehead (see, e.g., p.419 in [43]), there exists a triangulation $\tau$ of $|N(\omega)|$ finer than the cover $\alpha$. Let us choose a (possibly iterated) star refinement $\omega^{*}$ of $\omega$, the locally finite polyhedron $P_{1}=\left|N\left(\omega^{*}\right)\right|$ and continuous mappings $s_{1}: X \rightarrow P_{1}, r_{1}: P_{1} \rightarrow X$ such that $r_{1} \circ s_{1}$ and $\operatorname{id}_{X}$ are $\omega^{*}$-homotopic, and let us consider the cover $\alpha^{*}=r_{1}^{-1}\left(\omega^{*}\right)$ of $P_{1}$. All of the above can be chosen in such a way that:

(1) $P_{2}=\left|N\left(\alpha^{*}\right)\right|$ is a subpolyhedron (with the same set of vertices) of $P_{1}$ which is in turn a subpolyhedron of $(|N(\omega)|, \tau)$, and

(2) there are mappings $s_{2}: P_{2} \rightarrow P_{1}, r_{2}: P_{1} \rightarrow P_{2}$ with $r_{2} \circ s_{2}$ and $\operatorname{id}_{P_{1}}$ being $\alpha^{*}$-homotopic, and finally,

(3) the cover $\alpha^{\prime}=r_{2}^{-1}\left(\alpha^{*}\right)$ of $P_{2}$ refines the trace of the cover $\alpha$ on $P_{2}$.

Let $P=P_{2}$. It is clear that $P \omega$-homotopy dominates $X$, and that the mappings $\operatorname{id}_{P}, s_{2} \circ$ $s_{1} \circ r_{1} \circ r_{2}: P \rightarrow P$ being $\alpha^{\prime}$-near are homotopic. Note that by construction, the mappings $r_{1} \circ r_{2} \circ s_{2} \circ s_{1}$ and id $X$ are homotopic. By the homotopy invariance of the Lefschetz number, $\mathcal{E}(X)=\lambda\left(\operatorname{id}_{X}\right)=\lambda\left(r_{1} \circ r_{2} \circ s_{2} \circ s_{1}\right)$ and $\mathcal{E}(P)=\lambda\left(\operatorname{id}_{P}\right)=\lambda\left(s_{2} \circ s_{1} \circ r_{1} \circ r_{2}\right)$. It is well known (see, e.g., [41]) that, when defined for a pair of mappings $f$ and $g$, the Lefschetz numbers $\lambda(f \circ g)$ and $\lambda(g \circ f)$ are equal. Hence $\mathcal{E}(X)=\lambda\left(r_{1} \circ r_{2} \circ s_{2} \circ s_{1}\right)=\lambda\left(s_{2} \circ s_{1} \circ r_{1} \circ r_{2}\right)=\mathcal{E}(P)$.

Lemmas 11 and 14, together with the definition of approachability (Definition 1) imply the first purely topological fixed point property.

Theorem 15 Let $\mathcal{P}$ be the class of polyhedra, $X \in D(\mathcal{P})$ be a paracompact space with $\mathcal{E}(X) \neq 0$ and $\Gamma: X \rightrightarrows X$ be a u.s.c. approachable map with non-empty closed values. If $\Gamma$ is compact, then it has a fixed point. 
Proof By Lemma 14, for an arbitrarily chosen open cover $\omega$ of $X$, there exists a locally finite polyhedron $P$ with non-trivial Euler-Poincaré characteristic and a pair of continuous mappings $X \stackrel{s}{\rightarrow} P \stackrel{r}{\rightarrow} X$ such that, for each $x \in X,(r \circ s)(x)$ and $x$ are $\omega$-near. Consider the following commutative diagram:

$$
\begin{gathered}
X \stackrel{\Gamma}{\rightrightarrows} X \stackrel{s}{\rightarrow} P \\
r s \Gamma \uparrow \underset{\swarrow}{\swarrow} \quad \uparrow s \Gamma r . \\
X \underset{\Gamma}{\rightrightarrows} X \underset{s}{\rightarrow} P
\end{gathered}
$$

We show that the u.s.c. compact-valued map $s \Gamma r: P \rightrightarrows P$ is approachable in a stronger sense, sufficient for the existence of a fixed point. Being a compact subset of $P$, the set $K^{\prime}=\operatorname{cl}((s \Gamma r)(P))$ admits a cofinal family of open covers $\{\vartheta\}$. Any given arbitrary open cover $\vartheta$ of $K^{\prime}$, has a uniform refinement of the form $\left\{W\left[p_{i}\right]: p_{i} \in K^{\prime}, i=1, \ldots, n\right\}$, where $W$ is an entourage of a uniformity structure generating a topology equivalent to the initial $\mathrm{CW}$ topology on $P$. In addition, the entourage $W$ can be chosen small enough so as to satisfy the following: $W\left[K^{\prime}\right] \subset O$, where $O$ is an open neighborhood of a compact $A N R C$ such that $K^{\prime} \subset C \subset O \subset P$ provided by the main result (Theorem) in [38], with neighborhood retraction $\rho: O \rightarrow C$.

The map $s: \operatorname{cl}(\Gamma(X)) \rightarrow P$ being continuous on the compact set $\operatorname{cl}(\Gamma(X))$ is uniformly continuous. Thus, there exists a member $V$ of a uniform structure $\mathcal{V}$ defining an equivalent topology on $X$ such that $s(V[x]) \subset W[s(x)], \forall x \in \operatorname{cl}(\Gamma(X))$. By hypothesis, according to (7) there exists a continuous $(V, V)$-approximative selection $f: X \rightarrow X$ of $\Gamma$, i.e.,

$$
f(x) \in V[\Gamma(V[x])], \quad \forall x \in X
$$

Thus, $\forall p \in P$,

$$
s(f(r(p))) \in s(V[\Gamma(V[r(p)])]) \subset W[s \Gamma(V[r(p)])] .
$$

The continuous mapping $s \circ f \circ r$ maps $P$ into the tubular open neighborhood $W\left[K^{\prime}\right]$ in $P$. Being a continuous compact single-valued mapping of a locally finite polyhedron with non-zero Euler-Poincaré characteristic, $\rho \circ s \circ f \circ r: P \rightarrow C \hookrightarrow P$ has a fixed point $p_{W}=(\rho \circ$ $s \circ f \circ r)\left(p_{W}\right) \in \rho\left(W\left[s \Gamma\left(V\left[r\left(p_{W}\right)\right]\right)\right]\right)$. Hence, $p_{W} \in W^{2}\left[s \Gamma\left(V\left[r\left(p_{W}\right)\right]\right)\right]$, i.e., $p_{W} \in W^{2}\left[s\left(y_{W}\right)\right]$ with $y_{W} \in \Gamma\left(x_{W}\right), x_{W} \in V\left[r\left(p_{W}\right)\right]$. By compactness, there exist nets $\left\{p_{W}\right\}$ converging to some $\bar{p} \in P, r\left(p_{W}\right) \rightarrow r(\bar{p})=\bar{x}$ in $X, x_{W} \rightarrow \bar{x}$ in $X, y_{W} \rightarrow \bar{y}$ in $\operatorname{cl}(\Gamma(X)), s\left(y_{W}\right) \rightarrow s(\bar{y})$ in $P$. Consequently, $\bar{p}=s(\bar{y}) \in s \Gamma(\bar{x})=s \Gamma r(\bar{p})$.

By commutativity of diagram (8), the map $r s \Gamma: X \rightrightarrows X$ also has a fixed point $x_{\omega}=r s\left(y_{\omega}\right)$, $y_{\omega} \in \Gamma\left(x_{\omega}\right)$ satisfying $\left\{x_{\omega}, y_{\omega}\right\}$ are $\omega$-near, i.e., $x_{\omega}$ is an $\omega$-fixed point for $\Gamma$. Since $\operatorname{cl}(\Gamma(X))$ is compact, Lemma 11 ends the proof.

The novelty in Theorem 15 is that compactness is on the map rather than the domain and in the use of simple homotopy and approximation methods (see, e.g., Theorem 3.21 in [5] for the case where $X$ is compact and $P$ is the class of finite polyhedra). Surely, the theorem could be obtained using Lefschetz theory and homological methods, but the methods used here are notably simpler. 
Corollary 16 Every compact u.s.c. approachable map with non-empty closed values $\Gamma$ : $X \rightrightarrows X$ of an ANR $X$ with $\mathcal{E}(X) \neq 0$ has a fixed point.

Corollary 16 is a significant improvement on the Kakutani-Himmelberg fixed point theorem and on the Borsuk fixed point theorem for ARs. It holds true if the values of $\Gamma$ are $\infty$-proximally connected in the ANR $X$ (or $\Gamma$ admits a decomposition as in Example 10), thus extending the main theorem in [37] whereby $X$ is a compact ANR.

\section{Solvability results for $\mathrm{CNI}$ and applications}

The main solvability results for the convex as well as the non-convex CNI problem are presented in this section together with applications to QVI, MCP, and GOCP.

\subsection{CNI with quasiconvex objectives}

For simplicity, we start with CNI in the case of a convex domain and a convex-valued constraint $\Psi$ by extending the main result in [10] to non-compact domains.

Theorem 17 Let $X, Y$ be non-empty convex subsets in locally convex spaces $E, F$ and let:

(i) $\Phi: X \rightrightarrows Y$ be a compact u.s.c. map with non-empty closed values such that the restriction $\left.\Phi\right|_{\operatorname{conv}\{N\}}$ is approachable for each finite subset $N$ of $X$;

(ii) $\Psi: X \rightrightarrows X$ be a compact l.s.c. map with non-empty closed (hence compact) convex values; and

(iii) $\varphi: X \times Y \times X \rightarrow(-\infty,+\infty]$ be a continuous extended proper real function with $\varphi(u, y, \cdot)$ quasiconvex on $\Psi(u), \forall(u, y) \in X \times Y$.

Then CNI is solvable, i.e.,

$$
\left\{\begin{array}{l}
\exists \hat{u} \in \Psi(\hat{u}), \exists \hat{y} \in \Phi(\hat{u}), \text { with } \\
\varphi(\hat{u}, \hat{y}, \hat{u}) \leq \varphi(\hat{u}, \hat{y}, v), \forall v \in \Psi(\hat{u}) .
\end{array}\right.
$$

Proof Define the marginal map $M: X \times Y \rightrightarrows X$ by putting, for $(u, y) \in X \times Y$,

$$
M(u, y):=\left\{v \in \Psi(u) ; \varphi(u, y, v)=\min _{w \in \Psi(u)} \varphi(u, y, w)\right\} .
$$

The compactness and convexity of the values of $\Psi$, together with the lower semicontinuity of $\varphi$ and its quasi-convexity in the third argument when restricted to $\Psi(u)$, imply that $M$ has non-empty convex compact values. We verify that $M$ is u.s.c. To do this, in view of the fact that both $\Phi$ and $\Psi$ are compact maps, it suffices to verify that the graph of $M$ is closed (indeed, a compact map with closed graph is $u$.s.c.). To do this, let $\left(u_{\alpha}, y_{\alpha}, v_{\alpha}\right)_{\alpha}$ be a net in graph $(M)$ converging to $(u, y, v) \in X \times Y \times X$. Then,

$$
\begin{aligned}
\varphi(u, y, v) & \leq \liminf \varphi\left(u_{\alpha}, y_{\alpha}, v_{\alpha}\right)=\liminf \inf _{\alpha \in \Psi\left(u_{\alpha}\right)} \varphi\left(u_{\alpha}, y_{\alpha}, w\right) \\
& \leq \lim \sup _{\alpha} \inf _{w \in \Psi\left(u_{\alpha}\right)} \varphi\left(u_{\alpha}, y_{\alpha}, w\right) \leq \inf _{w \in \Psi(u)} \varphi(u, y, w),
\end{aligned}
$$

where the first inequality above follows from the lower semicontinuity of $\varphi$ and the last inequality from the upper semicontinuity of the marginal functional $\inf _{w \in \Psi(\cdot)} \varphi(\cdot, \cdot, w)$ (this upper semicontinuity follows at once from the facts that $\varphi$ is an upper semicontinuous functional and $\Psi$ is an l.s.c. set-valued $\left.\operatorname{map}^{\mathrm{r}}\right)$. Hence, $(u, y, v) \in \operatorname{graph}(M)$. 
Cellina's approximation theorem (Example 2 above) asserts that the restriction of the map $M$ to any compact subset of $X \times Y$ (in particular to finite convex polytopes) is approachable.

Define a map $\Gamma: X \times Y \rightrightarrows X \times Y$ by putting

$$
\Gamma(u, y):=M(u, y) \times \Phi(u), \quad(u, y) \in X \times Y .
$$

Now as a product map of u.s.c. approachable maps, the map $\Gamma$ is also u.s.c. and approachable on finite convex polytopes (and on compact subsets of its domain). It has non-empty compact values. Moreover, $\Gamma(X \times Y) \subset \Psi(X) \times \Phi(X) \subset K$ a compact subset in $X \times Y$. All conditions of Theorem 12 are thus satisfied. Therefore, $\Gamma$ has a fixed point $(\hat{u}, \hat{y}) \in \Gamma(\hat{u}, \hat{y})$, that is, $\hat{u} \in \Psi(\hat{u}), \hat{y} \in \Phi(\hat{u})$ and $\varphi(\hat{u}, \hat{y}, \hat{u}) \leq \varphi(\hat{u}, \hat{y}, v), \forall v \in \Psi(\hat{u})$.

\section{Remark 1}

(1) Theorem 3.1 in [10] corresponds to the case where, instead of the maps $\Phi, \Psi$ being compact, the space $X$ is compact and $\Psi$ is both u.s.c. and l.s.c.

(2) If in addition, $\forall u \in X$ with $u \in \Psi(u), \forall y \in \Phi(u)$ one has $\varphi(u, y, u) \geq 0$, then $\varphi(\hat{u}, \hat{y}, v) \geq 0, \forall v \in \Psi(\hat{u})$.

(3) If $\Psi(u)=X, \forall u \in X$, the continuity assumptions on $\varphi$ can be slightly relaxed to $\varphi$ is l.s.c. and $\varphi(\cdot, \cdot, u)$ is u.s.c.; in which case, Theorem 17 extends Theorem 1 in [44] to infinite dimensional spaces and to the case where $\Phi$ is a composition of convex as well as non-convex maps $\Phi$.

The map $\Psi$ may be a non-compact non-self map. In such a case, a compactness coercivity condition of the Karamardian type can be used to solve CNI (see, e.g., [45] for an early use in the context of variational inequalities).

Given two subsets $X$ and $C$ in a topological space $E$, denote by $\partial_{X}(C)=\operatorname{cl}(C) \cap \operatorname{cl}(X \backslash C)$ the boundary of $C$ relative to $X$, and by $\operatorname{int}_{X}(C)=C \cap\left(E \backslash \partial_{X}(C)\right)$ the interior of $C$ relative to $X$.

Theorem 18 Let $X, Y$ be non-empty convex subsets in locally convex spaces $E, F$, and $C$ be a non-empty compact convex subset of $X$. Let $\Phi: C \rightrightarrows Y$ and $\Psi: C \rightrightarrows X$ be maps satisfying:

(i) $\Phi$ is u.s.c. with non-empty compact values and approachable on convex finite polytopes in $C$;

(ii) the compression map $\Psi_{C}: C \rightrightarrows C$ defined by $\Psi_{C}(x):=\Psi(x) \cap C$ is l.s.c. with non-empty compact convex values;

(iii) $\varphi: C \times Y \times X \rightarrow(-\infty,+\infty]$ is an extended proper function continuous on $C \times Y \times C$ and with $\varphi(u, y, \cdot)$ convex on $\Psi(u), \forall(u, y) \in X \times Y$;

(iv) $\forall u \in C$ with $u \in \partial_{\Psi(u)}\left(\Psi_{C}(u)\right), \exists v \in \operatorname{int}_{\Psi(u)}\left(\Psi_{C}(u)\right)$ with $\varphi(u, y, v) \leq \varphi(u, y, u)$, $\forall y \in \Phi(u)$.

Then CNI is solvable.

Proof By Theorem 17, $\exists \hat{u} \in \Psi_{C}(\hat{u}), \exists \hat{y} \in \Phi(\hat{u})$ such that $\varphi(\hat{u}, \hat{y}, v) \geq \varphi(\hat{u}, \hat{y}, \hat{u}), \forall v \in \Psi_{C}(\hat{u})$. Given $v \in \Psi(\hat{u}) \backslash C$, two cases are possible.

Case 1: $\hat{u} \in \operatorname{int}_{\Psi(\hat{u})}\left(\Psi_{C}(\hat{u})\right)$. One can choose $0<\lambda<1$ small enough so that $w=\lambda v+$ $(1-\lambda) \hat{u} \in \Psi_{C}(\hat{u})$. Hence $\varphi(\hat{u}, \hat{y}, \hat{u}) \leq \varphi(\hat{u}, \hat{y}, w)$, and by convexity of $\varphi(\hat{u}, \hat{y}, \cdot)$ it follows that $\varphi(\hat{u}, \hat{y}, \hat{u}) \leq \lambda \varphi(\hat{u}, \hat{y}, v)+(1-\lambda) \varphi(\hat{u}, \hat{y}, \hat{u})$. Thus, $\varphi(\hat{u}, \hat{y}, \hat{u}) \leq \varphi(\hat{u}, \hat{y}, v)$. 
Case 2: $\hat{u} \in \partial_{\Psi(\hat{u})}\left(\Psi_{C}(\hat{u})\right)$. By (iv), $\exists \hat{v} \in \operatorname{int}_{\Psi(\hat{u})}\left(\Psi_{C}(\hat{u})\right)$ with $\varphi(\hat{u}, \hat{y}, \hat{v}) \leq \varphi(\hat{u}, \hat{y}, \hat{u})$. One can choose $0<\lambda<1$ small enough so that $w=\lambda v+(1-\lambda) \hat{v} \in \Psi_{C}(\hat{u})$. Hence $\varphi(\hat{u}, \hat{y}, \hat{u}) \leq$ $\lambda \varphi(\hat{u}, \hat{y}, v)+(1-\lambda) \varphi(\hat{u}, \hat{y}, \hat{v}) \leq \lambda \varphi(\hat{u}, \hat{y}, v)+(1-\lambda) \varphi(\hat{u}, \hat{y}, \hat{u})$, where the last inequality follows from (iv). Thus, $\varphi(\hat{u}, \hat{y}, \hat{u}) \leq \varphi(\hat{u}, \hat{y}, v)$ thus completing the proof.

Remark 2 Again, if $\Psi(u)=X, \forall u \in C$, the continuity assumptions on $\varphi$ can be slightly relaxed to $\varphi$ is l.s.c. and $\varphi(\cdot,,, w)$ is u.s.c., extending Theorem 1 in [44].

\subsection{CNI with non-convex objectives}

Non-convexity occurs naturally in optimization. For example it is well known that Paretooptimal sets in multi-objective programming are not necessarily convex. Rather, under suitable hypotheses on the objectives and constraints, they may be contractible retracts of the feasible set (see, e.g., [46]). Topological properties of solution sets of vector optimization have been extensively studied with central themes being compactness, (path) connectedness, contractibility, retractability, etc. (see, e.g., the works by Benoist [47] and Huy [48] and his group for a number of non-convex vector optimization settings).

Our goal in this section is to establish, based on the topological fixed point Theorem 15, topological solvability result for $\mathrm{CNI}$ involving functions whose sublevel sets are absolute retracts. Such functions are not unusual in non-convex optimization, as the example by Ricceri below suggests.

Example 19 ([49]) Given a closed convex subset $X$ in a Banach space $E$ and two functions $\phi, J: X \rightarrow \mathbb{R}$ such that:

(i) $\phi$ is l.s.c. convex such that $\exists v_{0} \in X$ with $\phi\left(v_{0}\right)=0$ and $\alpha:=\inf _{v \in X, v \neq v_{0}} \frac{\phi(v)}{\left\|v-v_{0}\right\|}>0$;

(ii) $J$ is Lipschitzian with constant $L<\alpha$.

Then each non-empty sublevel set of $\phi+J$ is an AR.

Indeed, given $\lambda \in \mathbb{R}$ such that $\{v \in X:(\phi+J)(v) \leq \lambda\}$ is non-empty, define $F: X \stackrel{\lambda-J}{\rightarrow} \mathbb{R} \stackrel{\Phi}{\rightarrow} X$ as $F(v):=\Phi(\lambda-J(v))$, where

$$
\Phi(t)= \begin{cases}\left.\left.\phi^{-1}(]-\infty, t\right]\right) & \text { if } t \geq 0, \\ \left\{v_{0}\right\} & \text { if } t<0,\end{cases}
$$

and observe that $\{v \in X:(\phi+J)(v) \leq \lambda\}=\operatorname{Fix}(F)$. It is easy to verify that $F$ is a set-valued contraction with closed convex values. It is well known that the fixed point set of such maps is an absolute retract. Similar arguments based on the topological structure of fixed points sets of set-valued maps can be used to construct other examples of functions whose level sets are retracts of sorts.

We shall now substantially weaken the convexity assumptions in Theorem 17 . Note first that if $\Psi$ and $\varphi$ are as in Theorem 17 (e.g., $\Psi$ has convex values and $\varphi$ is quasiconvex in its third argument), then for all $(u, y) \in X \times Y$, the subset $\arg \min _{\Psi(u)} \varphi(u, y, \cdot)$ as well as the sublevel sets of $\varphi(u, y, \cdot)$ are convex sets, thus retracts of every convex set containing them, in particular of $\Psi(u)$.

Theorem 20 Let $X, Y$ be ANRs with $\mathcal{E}(X), \mathcal{E}(Y) \neq 0$ and let:

(i) $\Phi: X \rightrightarrows Y$ be an approachable compact u.s.c. map with non-empty closed values; 
(ii) $\Psi: X \rightrightarrows X$ be a compact l.s.c. map whose values are ARs;

(iii) $\varphi: X \times Y \times X \rightarrow(-\infty,+\infty]$ be a continuous proper real function.

Assume that for all $(u, y) \in X \times Y$ any one of the following conditions holds:

(iv) $)_{1} \arg \min _{\Psi(u)} \varphi(u, y, \cdot)$ is a retract of $\Psi(u)$; or

(iv) $)_{2}$ for any $n \in \mathbb{N}$ large, the sublevel set $S_{\varphi(u, y, \cdot) \leq}^{(n)}:=\{v \in \Psi(u): \varphi(u, y, v) \leq$ $\left.\min _{\Psi(u)} \varphi(u, y, \cdot)+1 / n\right\}$ is a retract of $\Psi(u)$; or

(iv) $)_{3}$ for any $n \in \mathbb{N}$ large, for any $\epsilon>0$, there exists an $\epsilon$-deformation $h: S_{\varphi(u, y, \cdot) \leq}^{(n)} \times[0,1] \rightarrow S_{\varphi(u, y, \cdot) \leq}^{(n)}$ such that $h(\cdot, 1)$ can be extended to $\Psi(u)$.

Then CNI has a solution.

Proof The proof goes along the lines of that of Theorem 17. The constrained marginal map

$$
M(u, y):=\left\{v \in \Psi(u) ; \varphi(u, y, v)=\min _{w \in \Psi(u)} \varphi(u, y, w)\right\}
$$

$(u, y) \in X \times Y$ is $u$.s.c. with non-empty compact values for exactly the same reasons. Its values are precisely the sets $\arg \min _{\Psi(u)} \varphi(u, y, \cdot)$.

In case (iv) $)_{1}$ holds, being a retract of the compact absolute retract $\Psi(u)$, the set $\arg \min _{\Psi(u)} \varphi(u, y, \cdot)$ is also a compact AR, thus contractible. Therefore, the map $M$ is approachable by Example 4.

If (iv) ${ }_{2}$ holds, then the set $\arg \min _{\Psi(u)} \varphi(u, y, \cdot)$ can be written as a decreasing sequence

$$
\bigcap_{n=n_{0}}^{\infty}\left\{v \in \Psi(u): \varphi(u, y, v) \leq \min _{\Psi(u)} \varphi(u, y, \cdot)+1 / n\right\}
$$

of compact sublevel sets that are retracts of $\Psi(u)$. Such level sets are therefore themselves compact ARs, and the values $\arg \min _{\Psi(u)} \varphi(u, y, \cdot)$ of the map $M$ are compact $R_{\delta}$-sets in view of Example 6(iii) above. Thus, $M$ is approachable on the AR $X \times Y$ by Example 7(ii).

As of (iv) $)_{3}$, by an important result in the theory of retracts, it is necessary and sufficient for the closed subset $S_{\varphi(u, y,) \leq}^{(n)}$ of the absolute retract $\Psi(u)$ to be an AR as well (see, e.g., Lemma 5.6.3 in [31]).

In all three cases, as in the proof of Theorem 17, the map $\Gamma(u, y):=M(u, y) \times \Phi(u),(u, y) \in$ $X \times Y$, is u.s.c. and approachable. It has non-empty compact values. Moreover, $\Gamma(X \times Y) \subset$ $\Psi(X) \times \Phi(X) \subset K$ a compact subset in $X \times Y$. All conditions of Theorem 15 are thus satisfied (note that $\mathcal{E}(X \times Y)=\mathcal{E}(X) \times \mathcal{E}(Y) \neq 0$ ). Therefore, $\Gamma$ has a fixed point and the proof ends as in Theorem 17 .

Remark 3 Theorem 20 contains Theorem 3.1 of [50]. Indeed, recall that an ANR is contractible if and only if it is an AR. A compact AR is acyclic and has the fixed point property for single-valued continuous functions (Borsuk's theorem). In addition, condition (i) of Theorem 3.1 in [50] is relaxed. On the other hand, if the values of the map $\Phi$ are $\infty$ proximally connected (in particular contractible), then by Example 7 hypothesis (i) holds true.

As a particular case of Theorem 20, we have the following. 
Corollary 21 Let $X, Y, \Psi$, and $\varphi$ be as in Theorem 20, and let $\Phi: X \rightrightarrows Y$ be a map satisfying either one of the following conditions:

(a) $\Phi$ is a compact u.s.c. map with closed $\infty$-proximally connected values in $Y$.

(b) $\Phi$ admits a decomposition $\Phi(x)=\left(\Phi_{n} \circ \cdots \circ \Phi_{1}\right)(x)$, where each map $\Phi_{i}: X_{i-1} \rightrightarrows X_{i}$ is u.s.c. with $\infty$-proximally connected in an $A N R X_{i}$ for all $i=1, \ldots, n, X_{0}=X$, $X_{n}=Y$ and $X$ is compact.

Then problem CNI has a solution.

Proof It suffices to observe that since $X$ is an ANR, then by Examples 7(ii) and 10, $\Phi$ is approachable. The conclusion follows immediately from Theorem 20 .

The solvability results for CNI above apply to the various problems described in Section 2: namely, generalized quasi-variational inequalities QVI, variational-like inequalities of the Stampacchia type VIS, multivalued complementarity problems MCP, or general optimal control problem GOCP. The remainder of the paper is devoted to illustrating a few cases of applications. Space constraints impose limits on the discussion.

\subsection{Solving quasi-variational inequalities}

Consider QVI associated to the data $(X, Y, \Phi, \Psi, \theta, \eta, \phi)$ as defined in Section 2:

$$
\left\{\begin{array}{l}
\exists \hat{u} \in \Psi(\hat{u}), \exists \hat{y} \in \Phi(\hat{u}) \text { with } \\
\langle\theta(\hat{u}, \hat{y}), \eta(v, \hat{u})\rangle \geq \phi(\hat{u})-\phi(v), \forall v \in \Psi(\hat{u}),
\end{array}\right.
$$

where $X \subseteq E, Y \subseteq F, E$ being a vector space, $\left\langle F^{*}, F\right\rangle$ a dual pair of vector spaces, $\Psi: X \rightrightarrows X$, $\Phi: X \rightrightarrows Y, \theta: X \times Y \rightarrow F^{*}, \eta: X \times X \rightarrow F$ and $\phi: X \rightarrow \mathbb{R}$.

Particular instances of QVI were studied in [44, 45, 50-54], and many others. We refer to [17] and [21] for comprehensive discussions on the various aspects as well as the many applications of variational inequalities.

We shall apply now Corollary 21(a) and Theorem 17 to the functional $\varphi: X \times Y \times X \rightarrow \mathbb{R}$ given by $\varphi(u, y, v)=\langle\theta(u, y), \eta(v, u)\rangle+\phi(v)$ to obtain the following results.

Theorem 22 Given $X$ a convex subset of the normed space $E$ and $Y$ an $A N R$ with $\mathcal{E}(Y) \neq 0$ imbedded in the normed space $F$, let:

(i) $\Phi: X \rightrightarrows Y$ be a compact u.s.c. map with closed $\infty$-proximally connected values in $Y$;

(ii) $\Psi: X \rightrightarrows X$ be a compact l.s.c. map with closed convex values;

(iii) $\phi$ be continuous and convex and verify $\forall u \in X, \exists v_{u} \in \Psi(u)$ with $\phi\left(v_{u}\right)=0$ and

$$
\alpha_{u}=\min _{v \in \Psi(u), v \neq v_{u}} \frac{\phi(v)}{\left\|v-v_{u}\right\|}>0
$$

(iv) $\eta$ be continuous and $\forall u \in X, \eta(\cdot, u)$ be Lipschitzian with constant $L_{u}>0$;

(v) $\theta$ be continuous and $\forall(u, y) \in X \times Y,\|\theta(u, y)\|<\frac{\alpha_{u}}{L_{u}}$.

Then QVI has a solution.

Proof Observe that $\varphi(u, y, v)=J(v)+\phi(v)$ with $J(v)=\langle\theta(u, y), \eta(v, u)\rangle$ Lipschitzian with constant $\|\theta(u, y)\| L_{u}$. By Example 19 applied to a convex compact (hence complete) set $\Psi(u)$, the level sets of $\varphi(u, y, \cdot)$ restricted to $\Psi(u)$ are absolute retracts. Thus, all hypotheses of Theorem 20 including (iv) 2 are satisfied (a convex set in a normed spaces is an AR by the Dugundji's extension theorem). This ends the proof as QVI is a particular case of CNI. 
Suppose now that $X$ is a subset in a normed space $E$, and let, for a given $\rho>0, X_{\rho}$ be the set $X \cap D_{\rho}$, where $D_{\rho}$ is the closed disk of radius $\rho$ centered at the origin in $E$. Assuming that $X_{\rho}$ is non-empty, denote by $\Psi_{\rho}$ the compression of $\Psi$ restricted to $X_{\rho}$ given by $\Psi_{\rho}(u):=\Psi(u) \cap D_{\rho}, u \in X_{\rho}$. An immediate application of Theorem 18 is the following solvability result with a coercivity condition in lieu of the compactness of the set $X$.

Theorem 23 Consider QVI for the data $(X, Y, \Phi, \Psi, \theta, \eta, \phi)$ with $X$ being a non-empty convex subset of a normed space $E$ and $Y$ a non-empty convex subset of a locally convex space $F$, and $\theta$ and $\eta$ being continuous. Assume that:

(i) $\langle\theta(u, y), \eta(u, u)\rangle \geq 0, \forall(u, y) \in \operatorname{graph}(\Phi)$;

(ii) $\forall(u, p) \in X \times F^{*},\langle p, \eta(\cdot, u)\rangle$ is convex on $\Psi(u)$.

Assume, furthermore, that $\exists \rho_{0}>0$ such that $\forall \rho \geq \rho_{0}$ :

(iii) $X_{\rho}$ is compact non-empty and the map $\Psi_{\rho}$ is l.s.c. and has non-empty compact convex values on $X_{\rho}$;

(iv) the restriction of the map $\Phi$ to $X_{\rho}$ admits a decomposition as a finite composition of u.s.c. maps with non-empty compact $\infty$-proximally connected values through a sequence of ANRs;

(v) $\phi$ is convex and its restriction to $X_{\rho}$ is continuous;

(vi) $\forall u \in \Psi(u),\|u\|=\rho, \exists v \in \Psi(u),\|v\|<\rho$ with

$$
\max _{y \in \Phi(u)}\langle\theta(u, y), \eta(v, u)\rangle \leq \phi(u)-\phi(v)
$$

Then problem QVI has a solution.

Proof Take $C=X_{\rho}=X \cap D_{\rho}$ and $\varphi(u, y, v)=\langle\theta(u, y), \eta(v, u)\rangle+\phi(v)$ in Theorem 18.

\section{Remark 4}

(1) Let $\phi \equiv 0$. If there exists $v_{0} \in \bigcap_{u \in X} \Psi(u)$ such that

$$
\lim _{\|u\| \rightarrow \infty, u \in \Psi(u)} \max _{y \in \Phi(u)}\left\langle\theta(u, y), \eta\left(v_{0}, u\right)\right\rangle<0
$$

then hypothesis (vi) is satisfied. We thus obtain a generalization of a result in [50].

(2) It is easy to verify that an alternative coercivity condition to (vi) is:

(iv) $^{\prime}$ there exists a non-empty compact convex subset $C$ of $X$ such that

$$
\forall u \in X \backslash C, \exists v \in X \text { with } \max _{y \in \Phi(u)}\langle\theta(u, y), \eta(v, u)\rangle<\phi(u)-\phi(v) .
$$

(3) If $E=F$ and $\eta(v, u)=v-u$, then hypotheses (i)-(ii) are obviously satisfied. If $\eta(u, u)=0, \forall u \in X$, then (i) is obviously satisfied. However, it may happen that $\eta$ is not identically zero on the diagonal of $X \times X$ and yet problem QVI has a solution (see, e.g., [52]).

Note that given any subset $X$ of a normed space $E, X_{\rho}=X \cap D_{\rho}$ is a retract of $X$ (because $D_{\rho}$ is a retract of $E$ ). In our next result, we shall assume more, namely that $X_{\rho}$ is a deformation retract of $X$ (thus has the same homotopy type as $X$ ). 
Corollary 24 Assume that $X$ and $Y$ are ANRs in normed spaces with $\mathcal{E}(Y) \neq 0$, and let $\Phi: X \rightrightarrows Y$ be a map admitting a decomposition as a finite composition of u.s.c. maps with non-empty compact $\infty$-proximally connected values through a sequence of ANRs. Assume also that $\theta$ and $\eta$ are continuous and verify:

(i) $\langle\theta(x, y), \eta(x, x)\rangle \geq 0, \forall(x, y) \in \operatorname{graph}(\Phi)$.

Assume that $\exists \rho_{0}>0$ such that $\forall \rho \geq \rho_{0}$ :

(ii) $X_{\rho}$ is a compact deformation retract of $X$ and $\mathcal{E}(X) \neq 0$ (or more generally $X_{\rho}$ is compact and $\left.\mathcal{E}\left(X_{\rho}\right) \neq 0\right)$;

(iii) $\Psi_{\rho}$ is continuous with non-empty compact values;

(iv) $\forall(u, y) \in X_{\rho} \times Y$, the marginal set

$$
M(u, y)=\left\{v \in \Psi_{\rho}(u) ;\langle\theta(u, y), \eta(v, u)\rangle=\inf _{w \in \Psi_{\rho}(u)}\langle\theta(u, y), \eta(w, u)\rangle\right\}
$$

is $\infty$-proximally connected in $X_{\rho}$.

Then:

(1) QVI associated to the data $\left(X_{\rho}, Y, \Psi_{\rho}, \Phi, \theta, \eta, \phi\right)$ has a solution $u_{\rho}, \forall \rho \geq \rho_{0}$;

(2) if the set $\left\{u_{\rho}\right\}_{\rho \geq \rho_{0}}$ has a cluster point, then problem $Q V I$ has a solution.

Proof For $\rho \geq \rho_{0}$, since $X_{\rho}$ is a deformation retract of the ANR $X$, it is a compact ANR with $\mathcal{E}\left(X_{\rho}\right) \neq 0$ (the Euler-Poincaré characteristic being a homotopy invariant). Conclusion (1) readily follows from a general formulation of Theorem 20, whereby the marginal map $M: X_{\rho} \times Y \rightrightarrows X_{\rho}$ is u.s.c. and approachable (Example 7). Assume now that the set $\left\{u_{\rho}\right\}$ of solutions to the problems $Q V I\left(X_{\rho}, Y, \Psi_{\rho}, \Phi, \theta, \eta\right)$ has a subsequence $\left\{u_{\rho_{n}}\right\}_{n}$ converging to $\hat{u} \in X$ (an ANR is a closed set). For each $n, u_{\rho_{n}} \in \Psi_{\rho_{n}}\left(u_{\rho_{n}}\right)$ and for some $y_{n} \in \Phi\left(u_{\rho_{n}}\right)$, $\left\langle\theta\left(u_{\rho_{n}}, y_{n}\right), \eta\left(v, u_{\rho_{n}}\right)\right\rangle \geq 0, \forall v \in \Psi_{\rho_{n}}\left(u_{\rho_{n}}\right)$. Since for any large $\rho$, $\Psi_{\rho}$ is $u$ u.s.c. with closed values, it follows that $\hat{u} \in \Psi(\hat{u})$. Furthermore, the sequence $\left\{y_{n}\right\}$ being contained in the compact set $\Phi\left(\left\{u_{\rho_{n}}\right\} \cup\{\hat{u}\}\right)$ has a cluster point $\hat{y} \in \Phi(\hat{u})$. The continuity of $\theta$ and $\eta$ implies that $\langle\theta(\hat{u}, \hat{y}), \eta(v, \hat{u})\rangle \geq 0, \forall v \in \Psi(\hat{u})$.

Corollary 24 generalizes Theorem 3.8 of [50] in several ways.

Theorems 22, 23 and Corollary 24 for the solvability of QVI can be applied to generalize results by Isac and the second author [10] for QVIs involving monotone maps in a generalized sense defined on neighborhood retracts including Riemannian manifolds. This is the object of a subsequent work.

\subsection{Multivalued complementarity problem}

Recall that given a dual pair of vector spaces $\langle F, E\rangle$ and a cone $X \subset E$ with dual cone $X^{*}=$ $\{y \in F ;\langle y, x\rangle \geq 0, \forall x \in X\}$, and given a set-valued map $\Phi: X \rightrightarrows F$, a mapping $f: X \times F \rightarrow F$ and a functional $\phi: X \rightarrow \mathbb{R}$, the multivalued complementarity problem MCP (associated to $(X, \Phi, f, \phi))$ is

$$
\left\{\begin{array}{l}
\exists \hat{u} \in X, \exists \hat{y} \in \Phi(\hat{u}) \cap X^{*} \text { such that } \\
f(\hat{u}, \hat{y}) \in X^{*} \text { and }\langle f(\hat{u}, \hat{y}), \hat{u}\rangle=\phi(\hat{u}) .
\end{array}\right.
$$

The classical generalized multivalued complementarity problem corresponds to $\phi(u)$ being identically zero and $f(u, y)=y$ (see, e.g., [17]). 
We formulate a typical existence result for MCP that generalizes to non-convex maps classical results in [45] and their generalizations. Their proofs are similar to those presented there for convex-valued $\Phi$ and are left to the reader.

Theorem 25 Assume that $\Phi$ is u.s.c. with non-empty compact $\infty$-proximally connected values and that $\phi: X \rightarrow(-\infty, 0]$ is an l.s.c. convex functional. Assume also that there exists a compact convex subset $C$ of $X$ with non-empty interior relative to $X$ such that for each $u \in \partial_{X}(C)$ there exists $v \in \operatorname{int}_{X}(C)$ with $\inf _{y \in \Phi(u)}\langle y, u-v\rangle \geq \phi(v)-\phi(u)$.

Then:

(1) MCP has a solution provided $\phi(0)=0$ and $\phi(\lambda u)=\lambda \phi(u), \forall(\lambda, u) \in[1,+\infty) \times X$.

(2) $\exists \hat{u} \in C, \exists \hat{y} \in \Phi(\hat{u}) \cap X^{*}$ with $0 \leq\langle\hat{y}, \hat{u}\rangle \leq-\phi(\hat{u})$ provided $\phi(0)=0$ and $\phi(u+v) \leq \phi(u), \forall(u, v) \in X \times X$.

As an example of a by-product of Theorem 25, we obtain a generalization of known results that could be applied to finding stationary points of the Kuhn-Tucker type for nonsmooth programming problems with general objective functions. Assume that $E=\mathbb{R}^{n}$, $X=\mathbb{R}_{+}^{n}$, and $\phi: X \rightarrow(-\infty, 0]$ is an l.s.c. convex functional with $\phi(0)=0$ and $\phi(\lambda u)=\lambda \phi(u)$, $\forall(\lambda, u) \in[1,+\infty) \times X$. Let $g$ be a locally Lipschitz real function on $X$, and let us assume that $\Phi(u):=h(\partial f(u))$ is a homeomorphic image, lying in $X$, of the Clarke generalized gradient [51] of $g$ at $u$ (such a mapping $\Phi$ is of course u.s.c. and has non-empty compact contractible values). If there exist a constant $\beta>0$ and a vector $d \in X$ such that

$$
\begin{aligned}
& \forall u \in\{u \in X ;\langle d, u\rangle=\beta\}, \exists v \in\{u \in X ;\langle d, u\rangle<\beta\}, \\
& \text { with } \min _{y \in \Phi(u)}\langle y, u-v\rangle \geq \phi(v)-\phi(u),
\end{aligned}
$$

then, with $C:=\{u \in X ;\langle d, u\rangle \leq \beta\}$ (a compact set), one immediately obtains the solvability of MCP. Note that our coercivity condition above is independent of the mapping $f$ (which could be of the form $f(u, y):=M u+y+r, M \in \mathbb{R}^{n \times n}, r \in \mathbb{R}^{n}$, as in [54], or not).

Corollary 26 Let $X$ be a closed convex cone in $\mathbb{R}^{n}$, and let $\Phi: X \rightrightarrows \mathbb{R}^{n}$ be such that for any compact convex subset $C$ of $X$, the restriction $\left.\Phi\right|_{C}$ is compact-valued u.s.c. and approachable. Assume that $f(u, y)=y, \phi=0$, and that $\exists \alpha>0$ such that $\langle y-z, u\rangle \geq \alpha\|u\|^{2}$, $\forall(u, y) \in \operatorname{graph}(\Phi), \forall z \in \Phi(0)$. Then MCP has a solution.

\section{Generalized variational inequalities and co-equilibria on Lipschitzian ANRs}

The last section of this work establishes the existence of a solution for generalized variational inequalities as a co-equilibrium for an upper hemicontinuous non-self map with convex values defined on a Lipschitzian ANR.

Recall that, given a closed subset $X$ of a normed space $E$, an element $x_{0} \in X$ is an equilibrium for a set-valued map $\Phi: X \rightrightarrows E$ if $0 \in \Phi\left(x_{0}\right)$ (i.e., $x_{0}$ is a zero for $\Phi$ ). Naturally, such solvability theorems are always subject to tangency boundary conditions. In the absence of convexity, concepts of tangent and normal cones of non-smooth analysis are required. We briefly recall few facts about the contingent and circatangent cones (see, e.g., Mordukhovich [55], Aubin-Frankowska [56], Aubin-Cellina [57]). 


\section{Definition 27}

(i) The Bouligand-Severi contingent cone $T_{X}(x)$ to $X$ at $x$ is the upper limit in the sense of Painlevé-Kuratowski when $t \downarrow 0$ of the family $\left\{\frac{1}{t}(X-x)\right\}_{t>0}$.

(ii) The Clarke circatangent tangent cone $T_{X}^{C}(x)$ is the lower limit (i.e., the set of all limit points) when $t \downarrow 0$ and $x^{\prime} \rightarrow_{X} x$ of the family $\left\{\frac{1}{t}\left(X-x^{\prime}\right)\right\}_{t>0, x^{\prime} \in X}$.

A useful characterization of the Clarke cone is

$$
T_{X}^{C}(x):=\left\{v \in E: d_{X}^{0}(x)(v)=0\right\}
$$

where $d_{X}^{0}(x)(v)$ is the Clarke directional derivative (see [58]) of the locally Lipschitzian distance function $x \mapsto \operatorname{dist}(x ; X)$ at $x$ in the direction $v^{\mathrm{s}}$.

$T_{X}^{C}(x)$ is a closed convex cone contained in the closed cone $T_{X}(x)$. At interior points of $X, T_{X}^{C}(x)=T_{X}(x)=E$, the whole space. If $X$ is locally convex at $x \in X$, then $T_{X}^{C}(x)=T_{X}(x)=$ $T_{X}^{R}(x)=\operatorname{cl}\left(\bigcup_{t>0} \frac{1}{t}(X-x)\right)$ the tangent cone of convex analysis.

Definition 28 The set $X$ is said to be sleek at a point $x \in X$ if the set-valued map $x \mapsto T_{X}(x)$ is l.s.c. at $x$. $X$ is sleek if it so at each of its points.

If $X$ is sleek at $x$, then $T_{X}^{C}(x)=T_{X}(x)$ (hence, $X$ is regular at $x$ ), both cones being convex and closed cones; moreover, the Clarke's normal cone $N_{X}^{C}(x)=T_{X}^{C}(x)^{-}=\left(\partial^{0} \operatorname{dist}(x ; X)^{-}\right)^{-}=$ $\operatorname{cl}\left(\bigcup_{\lambda>0} \lambda \partial^{0} \operatorname{dist}(x ; X)\right)$, where $\partial^{0}$ is the Clarke's generalized gradient. Most importantly:

Proposition 29 If $X$ is sleek, then the map $N_{X}^{C}: X \rightrightarrows E^{\prime}$ has a closed graph and closed convex values.

The existence of an equilibrium is subject to the boundary condition of the BolzanoPoincaré-Halpern type being satisfied:

$$
\forall x \in \partial X, \quad \Phi(x) \cap T_{X}^{C}(x) \neq \emptyset .
$$

This tangency condition always implies a Ky Fan type condition expressed in terms of the normal cone $N_{X}^{C}(x)=T_{X}^{C}(x)^{-}$(the negative polar cone to $T_{X}^{C}(x)$ ):

$$
\forall x \in \partial X \quad\left(p \in N_{X}^{C}(x)\left(\text { or } p \in \partial^{0} \operatorname{dist}(x ; X)\right) \Longrightarrow \inf _{y \in \Phi(x)}\langle p, y\rangle \leq 0\right) .
$$

The reader is referred to [11] for a detailed discussion on equilibria for set-valued maps on non-smooth domains.

In view of the characterizations (3) and (4) of generalized variational inequalities, one introduces the following concept.

Definition 30 An element $x_{0} \in X$ is a co-equilibrium for $\Phi$ if it solves the generalized variational inequality $0 \in \Phi\left(x_{0}\right)-N_{X}^{C}\left(x_{0}\right)$.

\section{Remark 5}

(i) Clearly, an interior co-equilibrium is an equilibrium since, for such a point, $N_{X}^{C}=\{0\}$. 
(ii) Observe that $x_{0}$ is a co-equilibrium for $\Phi$ if and only if the maps $\Phi$ and $N_{X}^{C}$ coincide at $x_{0}$, i.e., $\Phi\left(x_{0}\right) \cap N_{X}^{C}\left(x_{0}\right) \neq \emptyset$. As $N_{X}^{C}\left(x_{0}\right)=T_{X}^{C}\left(x_{0}\right)^{-}$, this coincidence implies the infsup inequality $\inf _{y \in \Phi\left(x_{0}\right)} \sup _{v \in T_{X}^{C}(x)}\langle y, v\rangle \leq 0$.

(iii) Conversely, $\inf _{y \in \Phi\left(x_{0}\right)} \sup _{v \in T_{X}^{C}(x)}\langle y, v\rangle \leq 0$ implies that $x_{0}$ is a co-equilibrium for $\Phi$, provided $\Phi\left(x_{0}\right)$ is weakly compact. Indeed, the extended real-valued function $y \mapsto \sup _{v \in T_{X}^{C}(x)}\langle y, v\rangle$ is l.s.c. and convex, hence weakly l.s.c. Thus it achieves its infimum on $\Phi\left(x_{0}\right)$ at some $y_{0}$ verifying $\left\langle y_{0}, v\right\rangle \leq 0, \forall v \in T_{X}^{C}(x)$, i.e., $y_{0} \in N_{X}^{C}\left(x_{0}\right)$.

For simplicity, assume in this section, that the underlying space is a real Hilbert space $(E,\langle\cdot, \cdot\rangle)$ identified with its dual. ${ }^{\mathrm{t}}$ By a Hilbert space pair we mean a pair $(X, E)$ with $E$ a real Hilbert space and $X$ a closed subset of $E$. Recall that a map $\Phi$ is upper hemicontinuous on $X$ (u.h.c.) if for each $p \in E^{\prime}$, the support functional $x \mapsto \sigma_{\Phi(x)}(p)=\sup _{y \in \Phi(x)}\langle p, y\rangle$ is upper semicontinuous as an extended real-valued function on $X$. Always u.s.c. $\Longrightarrow$ u.h.c. The converse holds whenever $\Phi$ has convex weakly compact values. Define

$$
\begin{aligned}
& \mathbf{H}(X, E):=\{\Phi: X \rightrightarrows E: \Phi \text { is } u . h . c . \text { with non-empty closed convex values }\} \\
& \mathbf{H}_{\partial}(X, E):=\{\Phi \in \mathbf{H}(X, E): \Phi \text { verifies the boundary condition (10) }\}
\end{aligned}
$$

Definition 31 Let us say that a Hilbert space pair $(X, E)$ has the equilibrium property for the class $\mathbf{H}_{\partial}$ if and only if any map $\Phi \in \mathbf{H}_{\partial}(X, E)$ has an equilibrium in $X$.

Theorem 32 Assume that a Hilbert pair $(X, E)$ has the equilibrium property for the class $\mathbf{H}_{\partial}$. If $X$ is sleek, then any compact map $\Psi \in \mathbf{H}(X, E)$ has a co-equilibrium, i.e., $\exists x_{0} \in X$ solving the generalized variational inequality $0 \in \Psi\left(x_{0}\right)-N_{X}^{C}\left(x_{0}\right)$.

Proof The image $\Psi(X)$ of $\Psi$ is contained in a closed disk $D$ centered at the origin with radius $M>0$ in $E$. Consider the map $\Phi: X \rightrightarrows E$ given by $\Phi(x):=\Psi(x)-\left(N_{X}^{C}(x) \cap D\right)$. By Proposition 29 and since $X$ is sleek, the map $N_{X}^{C}: X \rightrightarrows E$ has a closed graph. The values $N_{X}^{C}(x) \cap D$ are closed, convex, and bounded, hence weakly compact. Thus, the map $x \mapsto$ $N_{X}^{C}(x) \cap D$ is u.h.c. with closed convex and bounded values. Being a linear combination of $u$.h.c. maps, $\Phi$ is also u.h.c. As the sum of a compact convex set and a closed bounded convex set, $\Phi(x)$ is closed and convex for each $x \in X$, i.e., $\Phi \in \mathbf{H}(X, E)$. We verify that $\Phi$ verifies the boundary condition (10). For any given $x \in \partial X$, since the cone $T_{X}^{C}(x)$ is closed and convex, by the Moreau decomposition theorem, any $y \in \Psi(x)$ has the form $y=y_{T}+y_{N}$ with $y_{T}=\operatorname{Proj}_{T_{X}^{C}(x)}(y)$ and $y_{N}=\operatorname{Proj}_{N_{X}^{C}(x)}(y),\left\langle y_{N}, y_{T}\right\rangle=0$. Therefore, $0=\left\langle y_{N}, y_{T}\right\rangle=\left\langle y_{N}, y-\right.$ $\left.y_{N}\right\rangle=\left\langle y_{N}, y\right\rangle-\left\|y_{N}\right\|^{2}$. By the Cauchy-Schwarz-Bunyakowsky inequality, $\left\|y_{N}\right\| \leq\|y\| \leq M$, that is, $y_{T}=y-y_{N} \in \Psi(x)-\left(N_{X}^{C}(x) \cap D\right)$, i.e., $\Phi(x) \cap T_{X}^{C}(x) \neq \emptyset$. The fact that $(X, E)$ has the equilibrium property for $\mathbf{H}_{\partial}$ ends the proof.

Recall that a subset $X$ of a metric space $(E, d)$ is an $L$-retract (of $E$ ) if there is a continuous neighborhood retraction $r: U \rightarrow X(U$ an open neighborhood of $X$ in $E$ ) and $L>0$ such that $d(r(x), x) \leq L \operatorname{dist}(x ; X)$ for all $x \in U$. An $L$-retract is clearly a neighborhood retract of $E$ and, in particular, is closed in $E$. The class of $L$-retracts is quite large and contains many subclasses of non-convex sets of interest in analysis and topology, e.g., closed subset of normed spaces that are bi-Lipschitz homeomorphic to closed convex sets, epi-Lipschitz subsets of normed spaces, prox-regular sets, etc. (see [59] and [11]). The following general 
variational inequality on $L$-retracts follows at once from Theorem 32 above and Theorem 5.3 in [59], which establishes that compact $L$-retracts belong to $\mathbf{H}_{\partial}(X, E)$.

Corollary 33 If $X$ is a compact $L$-retract in a Hilbert space $E$ with $\mathcal{E}(X) \neq 0$, and $\Psi \in$ $\mathbf{H}(X, E)$ is a compact-valued map, then $\Psi$ has a co-equilibrium.

Note that one can make use of a generalized Moreau decomposition theorem in Banach spaces to prove that Corollary 33 holds true in a Banach space $E$.

\subsection{Solvability for GOCP on compact epi-Lipschitz domains}

Given an interval $I$ in $\mathbb{R}$, a closed subset $X$ in a separable Banach space $E$, a map $F: I \times X \rightrightarrows$ $E$, let $S_{F}\left(t_{0}, u\right)$ be the solutions viable in $X$ for the Cauchy problem

$$
\left\{\begin{array}{l}
y^{\prime}(t) \in F(t, y(t)), \\
y\left(t_{0}\right)=u \in X,
\end{array} \quad t \in I, y(t) \in X\right.
$$

(assuming such solutions exist). Starting at a point $u \in X$, consider the journey along a trajectory $y(t)$ followed by a path to a point $v$ in a return set $\Psi(u) \subset X$. Assume that a cost $\varphi(u, y, v)$ is associated to this journey $\left(e . g ., \varphi(u, y, v)=\varphi_{1}(u, y)+\varphi_{2}(y, v)\right)$. We are interested in the particular control problem GOCP (see Section 2; we may assume with no loss in generality that $t_{0}$ is fixed)

$$
\left\{\begin{array}{l}
\exists \hat{u} \in X, \hat{u} \in \Psi(\hat{u}), \exists \hat{y} \in S_{F}(\hat{u}) \text { such that } \\
\varphi(\hat{u}, \hat{y}, \hat{u})=\inf _{v \in \Psi(\hat{u})} \varphi(\hat{u}, \hat{y}, v) .
\end{array}\right.
$$

Consider for an illustration the particular case treated in [60] where $F(t, y(t))=A y(t)+$ $R(t, y(t))$ with $A=\lim _{t \downarrow 0} \frac{1}{t}\left(U(t)-I d_{E}\right)$ being a closed densely defined linear operator which is the infinitesimal generator of a $C_{0}$-semigroup $\mathcal{U}=\{U(t)\}_{t \geq 0}$ of bounded linear operators on $E$ such that $\mathcal{U}(X) \subseteq X$. Let $R: I \times X \rightrightarrows E$ be a Carathéodory map ${ }^{\text {u }}$ with linear growth (i.e., $\sup _{z \in R(t, y)}\|y\| \leq \mu(t)(1+\|y\|)$ for some $\left.\mu \in L_{\text {loc }}^{1}(I, E)\right)$. The set of mild solutions $S_{F}\left(t_{0}, u\right)$ is the fixed point set of the composition

$$
C(I, E) \stackrel{N_{R}}{\rightrightarrows} L_{\mathrm{loc}}^{1}(I, E) \stackrel{M\left(t_{0}, u, \cdot\right)}{\longrightarrow} C(I, E)
$$

where $N_{R}(y):=\left\{f \in L_{\text {loc }}^{1}(I, E): f(t) \in R(t, y(t))\right.$ a.a. $\left.t \in I\right\}$ is the Nemetskij operator associated to $R$, and $M\left(t_{0}, u ; f\right)(t):=U\left(t-t_{0}\right) u+\int_{t_{0}}^{t} U(t-s) f(s) d s$ is the mild solution of the Cauchy problem $y^{\prime}(t) \in A y(t)+f(t), y\left(t_{0}\right)=u$. The solvability of GOCP is based on two crucial observations on the qualitative properties of the solution set of by Bothe [61] and Kryszewski [60].

\section{Theorem 34}

(i) ([61]) Assume that the semigroup $\mathcal{U}$ is compact and $R$ maps precompact subsets of $I \times X$ into compact sets in $E$. If the tangency condition with the Bouligand-Sever $i$ cone $R(t, y) \cap T_{X}(y) \neq \emptyset$ a.e. $t \in I$ for all $y \in X$ holds, then the map $S: I \times X \rightrightarrows C(I, X)$ given by $S\left(t_{0}, u\right)=S_{F}\left(t_{0}, u\right)$ is u.s.c. and has non-empty compact values. 
(ii) ([60]) If in addition $X$ is epi-Lipschitz in $E$, and the more restrictive tangency condition with the Clarke's cone $R(t, y) \cap T_{X}^{C}(y) \neq \emptyset$ for a.a. $t \in I$ and all $y \in X$ holds, then the values of the map $S$ are also $R_{\delta}$ sets.

These properties are set-valued generalizations to differential inclusions in infinite dimensions of Aronszajn's celebrated theorem on the $R_{\delta}$-set structure of the solution set of the classical single-valued Cauchy problem with continuous right-hand side [34]. They extend results by Plaskacz [62] where $X$ was a non-empty closed proximate retract ${ }^{\mathrm{v}}$ of $\mathbb{R}^{n}$. We conclude the paper with an extension of Theorem 4.1 in [10] for the solvability of GOCP.

Theorem 35 Assume that $X$ is a compact epi-Lipschitz set in a separable Banach space $E$ with $\mathcal{E}(X) \neq 0$ and that the above hypotheses on $F(t, y(t))=A y(t)+R(t, y(t))$ hold with the semigroup $\mathcal{U}$ being compact. If $\Psi: X \rightrightarrows X$ is l.s.c. with $A R$ values and $\varphi$ is continuous on $X \times C(I, E) \times X$, and quasiconvex with respect to the return variable $v$. Then GOCP has a solution provided $\Phi$ verifies the tangency condition

$$
R(t, y) \cap T_{X}^{C}(y) \neq \emptyset \quad \text { for a.a. } t \in I, \forall y \in X .
$$

Proof Apply Theorem 20 with $\Phi=S_{F}$ which is a u.s.c. compact approachable map by Example 7.

\section{Competing interests}

The authors declare that they have no competing interests.

\section{Authors' contributions}

All authors contributed equally to the writing of this paper. All authors read and approved the final manuscript.

\section{Author details}

'Department of Mathematics, King Abdulaziz University, P.O. Box 80203, Jeddah, 21589, Saudi Arabia. ${ }^{2}$ Department of Mathematics and Statistics, Brock University, Saint Catharines, Ontario L2S 3A1, Canada.

\section{Acknowledgements}

This work was funded by the Deanship of Scientific Research (DSR), King Abdulaziz University, Jeddah, under grant No. (130-127-D1435). The authors acknowledge with thanks DSR's technical and financial support.

a One could thus reasonably argue that variational inequalities go as far back as the establishment of optimality conditions for minimization problems, i.e., to Pierre de Fermat's necessary optimality condition for an equilibrium.

b Continuity: $\exists C>0,|a(u, v)| \leq C\|u\|\|v\|, \forall u, v \in E$. Coercivity: $a(u, u) \geq \alpha\|u\|^{2}, \forall u \in E$.

$c$ Using the Banach contraction principle presents a clear computational advantage of approximation by Picard iterations.

d A Ky Fan map has convex values and open pre-images. Boundedness of domain (thus weak compactness) follows from the coercivity of $a$.

e Based on Ky Fan's extension of the Knaster-Kuratowski-Mazurkiewicz principle to vector spaces of arbitrary dimension.

f That is a pair of real vector spaces $E, F$ together with a bilinear form $\langle\cdot, \cdot\rangle: F \times E \rightarrow \mathbb{R}$ such that $\forall x \in E \backslash\{0\}, \exists y \in F$ with $\langle y, x\rangle \neq 0$ and $\forall y \in F \backslash\{0\}, \exists x \in E$ with $\langle y, x\rangle \neq 0$.

9 A real function $f$ on a convex subset of a vector space is quasiconvex if $f(z) \leq \max \{f(x), f(y)\}$ for all $z \in[x, y]$. It is semi-strictly quasiconvex if $f(x)>f(y) \Longrightarrow f(x)>f(z)$ for all $z \in] x, y]$.

$h$ The strict inequality cannot be replaced by a large inequality as the quasiconvex differentiable function $f(x)=x^{3}$, $x \in[-1,1]$ indicates. The strict inequality can be replaced by the large inequality for the smaller class of differentiable pseudoconvex functions (which includes convex functions). In such a case, $[\exists \hat{u} \in X, \forall v \in X,\langle\nabla f(\hat{u}), v-\hat{u}\rangle \geq 0] \Longleftrightarrow f(\hat{u})=\min _{X} f(v)$.

i Note that if $f$ is semistrictly quasiconvex and I.s.c., then $c \mid\left(S_{f<}(u)\right)=S_{f \leq}(u)$ for all $u \in X \backslash \arg \min f$. Indeed, if $y \in S_{f \leq}(u)$ and $f(y)=f(u)$, consider $y_{1} \in X$ with inf $f \leq f\left(y_{1}\right)<f(y)=f(u)$. By semistrict quasi-convexity, $f\left(y_{i}\right)<f(y)=f(u)$ for any net $\left\{y_{i}\right\}$ converging to $y$ along the line segment $\left[y_{1}, y\right) \subset X$.
} 
j A map $\Phi: X \rightrightarrows Y$ of two topological spaces $X$ and $Y$ is said to be upper semicontinuous at a point $x_{0} \in X$ if for any open neighborhood $V$ of $\Phi\left(x_{0}\right)$ in $Y$, there exists an open neighborhood $U$ of $x_{0}$ in $X$ such that $\Phi(U) \subset V$. The map $\Phi$ is said to be upper semicontinuous (u.s.c. for short) on $X$ if it is upper semicontinuous at every point of $X$. Note that $\Phi$ is u.s.c. on $X$ if and only if the upper inverse image $\{x \in X ; \Phi(x) \subset V\}$ of any open subset $V$ of $Y$ is open in $X$.

${ }^{k}$ Note that since topological spaces are assumed to be Hausdorff, a neighborhood retract $A$ of $X$ is closed in $X$.

I The inclusions are strict. A Euclidean sphere is an ANR but not an $A R$. The set $\Gamma:=\left\{\left(x, \sin \left(\frac{1}{x}\right)\right) \in \mathbb{R}^{2}: 0<x \leq 1\right\} \cup\{(0, y):-1 \leq y \leq 1\}$ is an AANR but not an ANR (it is not locally contractible!).

$\mathrm{m}$ It is well known that all homology, cohomology, homotopy, and cohomotopy groups of an $A R$ are trivial. Also, every retract of an $A R$ is also an $A R$

$n$ An $R_{\delta}$ set is the intersection of a countable decreasing sequence of compact contractible metric spaces.

- A point $x_{\omega} \in X$ such that, for some $y_{\omega} \in \Phi\left(x_{\omega}\right)$, both $x_{\omega}$ and $y_{\omega}$ belong to a common member $W$ of $\omega$

$P$ For the definition of the nerve of a covering, see Definition 5.3, p.172 in Dugundji [43]. Given a topological space $Y$ and an open cover $\omega$ of $Y$, two mappings $f, g: X \rightarrow Y$ are said to be $\omega$-near if for each $x \in X,\{f(x), g(x)\} \subset W$ for some member $W$ of $\omega$. They are said to be $\omega$-homotopic if there exists a deformation $h: X \times[0,1] \rightarrow Y$ joining $f$ and $g$ satisfying $\exists W \in \omega$ with $h(\{x\} \times[0,1]) \subset W \forall x \in X$. If $Y$ is an $A N R$, every open cover $\omega$ of $Y$ admits a refinement $\alpha$ such that any two continuous mappings $f, g: X \rightarrow Y$ that are $\alpha$-near are $\omega$-homotopic (Lemma 7.2 in [42]).

q The Euler-Poincaré characteristic $\mathcal{E}(X)$ of a space $X$ is assumed to be a homotopy invariant. This is the case when $X$ is compact, with $\mathcal{E}(X)$ being the signed finite sum of Betti numbers $\sum_{i>0}(-1)^{i} \beta_{i}, \beta_{i}=\operatorname{dim} H^{i}(X ; \mathbf{Q})$, where the cohomology graded linear space $\left\{H^{i}(X ; \mathbf{Q})\right\}$ is of finite type. It turns out that, in this case, $\mathcal{E}(X)=\lambda(i \mathrm{id} X)$ the Lefschetz number of the identity mapping on $X$. A homotopically invariant Euler characteristic can be defined for large classes of non-compact spaces, e.g., finite unions of convex sets, non-compact complex algebraic varieties, $n$-dimensional hyperbolic Riemannian manifolds with finite volume, etc. (see, e.g., Chen [63], Gromov [64], and Harder [65]).

${ }^{r}$ If a set-valued map $\Psi$ is I.s.c. and a real function $f(u, w)$ is u.s.c., then the marginal function $g(u)=\inf _{w \in \Psi(u)} f(u, w)$ is u.s.c. (see [56]).

$s$ The mapping $v \mapsto d_{X}^{0}(x)(v)$ is finite, positively homogeneous, subadditive, and Lipschitz continuous on $E$. In addition, $(x, v) \mapsto d_{X}^{0}(X)(v)$ is u.s.c. on $X \times E$. The generalized gradient $\partial^{0}$ dist $(x ; X)$ is the convex weak*-convex set of linear forms $\left\{p \in E^{\prime}:\langle p, v\rangle \leq d_{X}^{0}(x)(v)\right\}$.

$\mathrm{t}$ The results below remain valid with a dual pair $\left(E, E^{\prime}\right)$ of a normed space and its topological dual.

$u$ That is, $R$ has convex values, is measurable in $t$ for all $y$, and is u.s.c. in $y$ for a.a. $t \in I$.

$\checkmark$ That is, there exists a continuous neighborhood retraction $r: U \rightarrow X$ with $r(x)=x, \forall x \in X$ and $\|r(x)-x\|=\operatorname{dist}(x, X)$, $\forall x \in U$.

\section{Received: 9 February 2015 Accepted: 17 May 2015 Published online: 12 June 2015}

\section{References}

1. Fichera, G: La nascita della teoria delle disequazioni variazionali ricordata dopo trent'anni. Accademia Nazionale dei Lincei 114, 47-53 (1995)

2. Hartmann, P, Stampacchia, G: On some non-linear elliptic differential-functional equations. Acta Math. 115, 271-310 (1966)

3. Brézis, H: Analyse fonctionelle, Théorie et applications. Masson, Paris (1983)

4. Latif, A, Luc, DT: Variational relation problems: existence of solutions and fixed points of set-valued contraction mappings. Fixed Point Theory Appl. 2013, 315 (2013)

5. Ben-El-Mechaiekh, H: Approximations and selections methods for set-valued maps. In: Al-Mezel, SAR, Al-Solamy, FRM, Ansari, QH (eds.) Fixed Point Theory, Variational Analysis, and Optimization, pp. 77-138. Chapman and Hall/CRC, Boca Raton (2014)

6. Minty, GJ: On variational inequalities for monotone operators I. Adv. Math. 30, 1-7 (1978)

7. Dugundji, J, Granas, A: KKM-maps and variational inequalities. Ann. Sc. Norm. Super. Pisa, Cl. Sci. (4) 5, 679-682 (1978)

8. Allen, G: Variational inequalities, complementarity problems and duality theorems. J. Math. Anal. Appl. 58, 1-10 (1977)

9. Lassonde, M: On the use of KKM multifunctions in fixed point theory and related topics. J. Math. Anal. Appl. 97, 151-201 (1983)

10. Ben-El-Mechaiekh, H, Isac, G: Generalized multivalued variational inequalities. In: Andreian-Cazacu, C, Letho, O, Rassias, TM (eds.) Topology and Analysis, pp. 115-142. World Scientific, River Edge (1998)

11. Ben-El-Mechaiekh, H: On nonlinear inclusions in non-smooth domains. Arab. J. Math. 1, 395-416 (2012)

12. Baiocchi, C, Capelo, A: Variational and Quasivariational Inequalities. Applications to Free-Boundary Problems. Wiley, New York (1984)

13. Kinderlehrer, D, Stampacchia, G: An Introduction to Variational Inequalities and Their Applications. Classics in Applied Mathematics, vol. 31. SIAM, Philadelphia (2000)

14. Nagurney, A: Network Economics: A Variational Inequality Approach. Academic Publishers, Dordrecht (1983)

15. Granas, A: Méthodes topologiques en analyse convexe. Séminaire de Mathématiques Supérieures, vol. 110. Les Presses de l'Université de Montréal, Montreal (1990)

16. Cottle, RW, Pang, JS, Stone, RE: The Linear Complementarity Problem. Academic Press, Boston (1992)

17. Isac, G: Complementarity Problems. Lecture Notes in Math., vol. 1528. Springer, Berlin (1992)

18. Murty, KG: Linear Complementarity, Linear and Nonlinear Programming. Heldermann, Berlin (1988)

19. Facchinei, F, Pang, JS: Finite Dimensional Variational Inequalities and Complementarity Problems, Vol. I and II. Springer, New York (2003)

20. Konnov, IV: Equilibrium Models and Variational Inequalities. Elsevier, Amsterdam (2007)

21. Ansari, QH, Lalitha, CS, Mehta, M: Generalized Convexity, Nonsmooth Variational Inequalities, and Nonsmooth Optimization. CRC Press, Boca Raton (2014)

22. Gwinner, J: On fixed points and variational inequalities - a circular tour. Nonlinear Anal. 5, 565-583 (1981) 
23. Blum, E, Oettli, W: From optimization and variational inequalities to equilibrium problems. Math. Stud. $63,123-145$ (1994)

24. Agarwal, RP, O'Regan, D: Nonlinear generalized quasi-variational inequalities: a fixed point approach. Math. Inequal. Appl. 6, 133-143 (2003)

25. Ansari, QH: An introduction to variational-like inequalities. In: Al-Mezel, SAR, Al-Solamy, FRM, Ansari, QH (eds.) Fixed Point Theory, Variational Analysis, and Optimization, pp. 207-245. Chapman and Hall/CRC, Boca Raton (2014)

26. Hadjisavvas, N: Convexity, generalized convexity and applications. In: Al-Mezel, SAR, Al-Solamy, FRM, Ansari, QH (eds.) Fixed Point Theory, Variational Analysis, and Optimization, pp. 139-171. Chapman and Hall/CRC, Boca Raton (2014)

27. Aussel, D: New developments in quasiconvex optimization. In: Al-Mezel, SAR, Al-Solamy, FRM, Ansari, QH (eds.) Fixed Point Theory, Variational Analysis, and Optimization, pp. 173-204. Chapman and Hall/CRC, Boca Raton (2014)

28. Ben-El-Mechaiekh, H, Deguire, P: Approximation of non-convex set-valued maps. C. R. Acad. Sc. Paris 312, 379-384 (1991)

29. Ben-El-Mechaiekh, H: Continuous approximation of set-valued maps, fixed points and coincidences. In: Florenzano, M, Guddat, J, Jimenez, M, Jongen, HT, Lagomasino, GL, Marcellan, F (eds.) Proceedings of the Second International Conference on Approximation and Optimization, pp. 69-97. Peter Lang, Frankfurt (1995)

30. Cellina, A: A theorem on the approximation of compact multivalued mappings. Atti Accad. Naz. Lincei, Rend. Cl. Sci. Fis. Mat. Nat. (8) 47, 429-433 (1969)

31. Van Mill, J: Infinite Dimensional Topology. North Holland, Amsterdam (1989)

32. Mas Colell, A: A note on a theorem of F. Browder. Math. Program. 6, 229-233 (1974)

33. McLennan, A: Approximation of contractible valued correspondences by functions. J. Math. Econ. 20, 591-598 (1991)

34. Aronszajn, N: Le correspondant topologique de l'unicité dans la théorie des équations différentielles. Ann. Math. 43, 730-738 (1942)

35. Dugundji, J: Modified Vietoris theorems for homotopy. Fundam. Math. LXVI, $223-235$ (1970)

36. Kryszewski, W: Graph-approximation of set-valued maps on noncompact domains. Topol. Appl. 83, 1-21 (1998)

37. Górniewicz, L, Granas, A, Kryszewski, W: On the homotopy method in the fixed point index theory of multivalued mappings of compact absolute neighborhood retracts. J. Math. Anal. Appl. 161, 457-473 (1991)

38. Girolo, J: Approximating compact sets in normed linear spaces. Pac. J. Math. 98, 81-89 (1982)

39. Fan, K: Fixed point and minimax theorems in locally convex topological linear spaces. Proc. Natl. Acad. Sci. USA 38 121-126 (1952)

40. Himmelberg, CJ: Fixed points for compact multifunctions. J. Math. Anal. Appl. 38, 205-207 (1972)

41. Granas, A: Points fixes pour les applications compactes: espaces de Lefschetz et la théorie de l'indice, vol. 68. Les Presses de l'Université de Montréal, Montreal (1980)

42. Dugundii, J: An extension of Tietze's theorem. Pac. J. Math. 1, 353-367 (1951)

43. Dugundji, J: Topology. Allyn and Bacon, Boston (1966)

44. Parida, J, Sen, A: A variational-like inequality for multifunctions with applications. J. Math. Anal. Appl. 124, 73-81 (1987)

45. Itoh, S, Takahashi, W, Yanagi, K: Variational inequalities and complementarity problems. J. Math. Soc. Jpn. 30, 23-28 (1978)

46. Slavov, ZD, Slavova Evans, C: Compactness, contractibility and fixed point properties of the Pareto sets in multi-objective programming. Appl. Math. 2, 556-561 (2011)

47. Benoist, J: Contractibility of the efficient set in strictly quasi-concave vector maximization. J. Optim. Theory Appl. 110 325-336 (2001)

48. Huy, NQ: Arcwise connectedness of the solution sets of a semistrictly quasiconcave vector maximization problem. Acta Math. Vietnam. 27, 165-174 (2002)

49. Ricceri, B: A class of functions whose sublevel sets are absolute retracts. Topol. Appl. 155, 871-873 (2008)

50. Yao, JC: The generalized quasi-variational inequality problem with applications. J. Math. Anal. Appl. 158, 139-160 (1991)

51. Chan, D, Pang, JS: The generalized quasi-variational inequality problem. Math. Oper. Res. 7, 211-222 (1982)

52. Dien, NH: Some remarks on variational-like and quasivariational-like inequalities. Bull. Aust. Math. Soc. 46, 335-342 (1992)

53. Gowda, MS, Pang, JS: Some existence results for multivalued complementarity problems. Math. Oper. Res. 17 657-670 (1992)

54. Parida, J, Sen, A, Kumar, A: A linear complementarity problem involving a subgradient. Bull. Aust. Math. Soc. 37, 345-351 (1988)

55. Mordukhovich, B: Variational Analysis and Generalized Differentiation, Vol. I and II. Springer, Berlin (2006)

56. Aubin, JP, Frankowska, H: Set-Valued Analysis. Birkhäuser, Boston (1990)

57. Aubin, JP, Cellina, A: Differential Inclusions. Springer, Berlin (1984)

58. Clarke, F: Optimization and Nonsmooth Analysis. Les publications CRM, Montréal (1989)

59. Ben-El-Mechaiekh, H, Kryszewski, W: Equilibria of set-valued maps on non-convex domains. Trans. Am. Math. Soc. 349, 4159-4179 (1997)

60. Kryszewski, W: Topological structure of solution sets of differential inclusions: the constrained case. Abstr. Appl. Anal. 6, 325-351 (2003)

61. Bothe, D: Multivalued differential equations on graphs and applications. Ph.D. thesis, Universität Paderborn (1992)

62. Plaskacz, S: On the solution sets for differential inclusions. Boll. Un. Math. Ital. A (7) 6-A, 387-394 (1992)

63. Chen, B: On the Euler characteristic of finite unions of convex sets. Discrete Comput. Geom. 10, 79-93 (1993)

64. Gromov, M: Volume and bounded cohomology. Publ. Math. IHÉS 56, 5-100 (1982)

65. Harder, G: A Gauss-Bonnet formula for discrete arithmetically defined groups. Ann. Sci. Éc. Norm. Super. 4, 409-455 (1971) 